
'Society for Makers, Artists, Researchers and Technologists (SMART)' is an American publishing house committed to publish high quality research articles which can bring value and momentum to current research in the domain of Electronics and Communication Technology and allied fields. As a part of this, it has started an international journal titled "American Journal of Electronics \& Communication".

"American Journal of Electronics \& Communication" (AJEC) is an open access, indexed, multidisciplinary peer reviewed journal that provides a platform for academicians and researchers all over the globe, to publish their research articles related to current trends and developments in Electronics and Communication Technology like microwave, semiconductor devices, VLSI, signal processing, communication, Internet of Things, Machine Learning, Artificial Intelligence etc. The main objective of AJEC is to cultivate innovative ideas among young minds and thus provide a proper guidance to the new frontiers and emerging trends in Electronics \& Communication Engineering.

The American Journal of Electronics \& Communication makes a maximum effort to publish submitted papers as quickly as possible. Primary reviews would be completed within two months. The authors are then requested to make necessary changes/improvements to their manuscripts within a span of two-three weeks. The whole review process fully respects standard Ethical Guidelines for Peer Reviewers. 


\section{American Journal of}

Electronics \& Communication

\section{Editor in Chief}

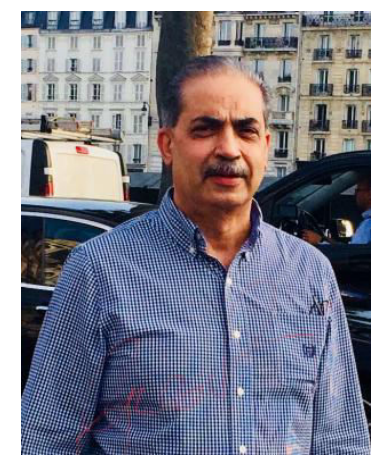

\section{Prof. Bob Gill}

British Columbia Institute of Technology

Research Interest: Sustainability, Sustainable Development, Renewable Energy Technologies, Power Generation, Energy Engineering, Energy Conversion, Energy Efficiency in Building Distributed Generation, Energy Saving Electricity, Energy Management, Energy Modeling, Solar Energy, Solar Radiation, Energy Utilization, Life-Cycle Assessment, Sustainable Energy, Alternative Energy, Clean Energy, Green Energy, Energy Modelling.

\section{Managing Editor}

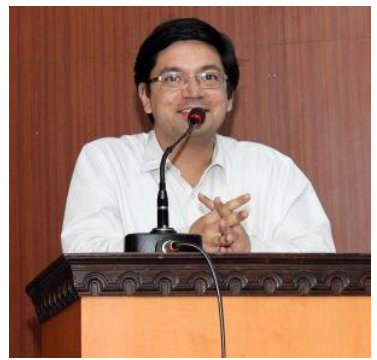

\section{Dr. Satyajit Chakrabarti}

Institute of Engineering \& Management

Research Interest: Machine Learning, IoT, Big Data Analytics, Data Mining, Algorithms, Robotics, Sensors, Human Computer Interface, Networking \& MANET, Wireless Communication.

\section{Dr. Malay Gangopadhyay}

Institute of Engineering \& Management

Research Interest: Microwave Technology, Antenna, IoT, Robotics, Wireless Communication. 


\section{Associate Editor}

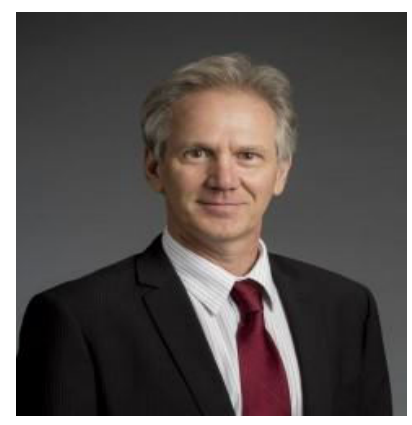

\section{Dr. Samuel Kozaitis}

Florida Institute of Technology

Research Interest: Development of algorithms to reduce noise in signals and images, magneto optic and ferroelectric materials, advanced signal processing algorithms, wavelet-based processing and higher-order correlations.

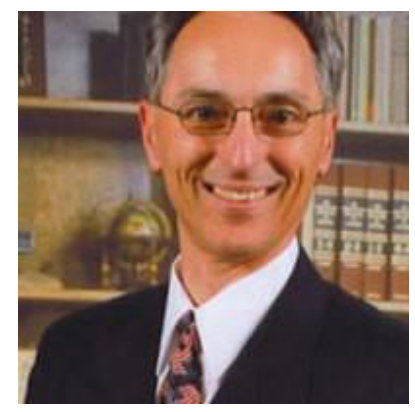

\section{Dr. Veton Kepuska}

Florida Institute of Technology

Research Interest: Neural networks, language modeling, digital signal processing, biometrics, adaptive filtering, telematics, speech recognition, speaker identification, pattern recognition, text to speech.

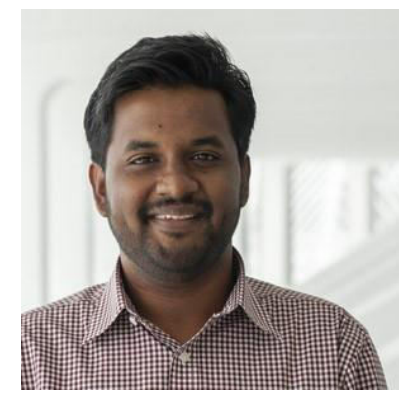

\section{Dr. Ashiq Adnan Sakib}

Florida Polytechnic University

Research Interest: Asynchronous Design, Advanced Digital Design, Low-power Design, and Formal Verification. 


\section{Contents}

\begin{tabular}{|c|c|c|c|}
\hline Sl. No. & Title of the Paper & Authors & $\begin{array}{l}\text { Name } \\
\text { Pages }\end{array}$ \\
\hline 1 & $\begin{array}{l}\text { A Survey on Robot Behavior and } \\
\text { Distance Estimation in } \\
\text { IndoorGML Maps Implementation }\end{array}$ & $\begin{array}{l}\text { Mohd Aaqib Lone, } \\
\text { Owais mujtaba khanday, } \\
\text { Aadil Ganie Gani }\end{array}$ & $1-7$ \\
\hline 2 & $\begin{array}{l}\text { Character Segmentation for } \\
\text { Handwritten Bangla } \\
\text { Words using Image Processing }\end{array}$ & $\begin{array}{l}\text { Poojarini Mitra, Kaustav } \\
\text { Bhattacharjee, Anirban Das, } \\
\text { Sayan Kumar Dey, Deepjyoti } \\
\text { Chakraborty, Aritra Ghosal, } \\
\text { Shadab Akhtar }\end{array}$ & $8-11$ \\
\hline 3 & $\begin{array}{l}\text { Android Controlled Home } \\
\text { Automation }\end{array}$ & Pankaj Kapoor, Suryadipta Sarkar & $12-16$ \\
\hline 4 & $\begin{array}{l}\text { Indian Sign Language Classification } \\
\text { (ISL) using Machine Learning }\end{array}$ & $\begin{array}{l}\text { Subhalaxmi Chakraborty, Nanak } \\
\text { Bandyopadhyay, Piyal } \\
\text { Chakraverty, Swatilekha Banerjee, } \\
\text { Zinnia Sarkar, Sweta Ghosh }\end{array}$ & $17-21$ \\
\hline 5 & $\begin{array}{l}\text { Fake Note Detection using Machine } \\
\text { Learning Techniques }\end{array}$ & $\begin{array}{l}\text { Subhalaxmi Chakraborty, } \\
\text { Soumyadip Banerjee, Biman } \\
\text { Kumar Singha, SayaniGhati }\end{array}$ & $22-29$ \\
\hline 6 & $\begin{array}{l}\text { Ticket Analytics by an AMS Team to } \\
\text { Assess Applications Deficiencies }\end{array}$ & Biswadeb Bandyopadhyay & $30-38$ \\
\hline
\end{tabular}




\title{
A Survey on Robot Behavior and Distance Estimation in IndoorGML Maps Implementation
}

\author{
Mohd Aaqib Lone, Owais mujtaba khanday, Aadil Ganie Gani (University of Miskolc, Hungary) \\ mohdaaqiblone@gmail.com
}

\begin{abstract}
The significance of behavior-based robots and the indoor navigation system has increased significantly over the past few years in its monitoring and wayfinding, taking into account the existence of robot behavior in various fields of social interest. The basic strategy of the overall behavior tasks in a behavior-based system are divided into smaller independent behaviors that focus on the performance of specific tasks such as the behavior of a robot for indoor environments. One behavior will concentrate on the wall, while the other focuses on avoiding obstacles, etc. Indoor navigation is the main concern since almost a lot of individuals spend more time in the indoor climate. In different fields such as hospitals, transportation, marketing, and military purposes, it becomes an essential thought. The main goal of this paper to give a survey of distance estimation in IndoorGML, behaviors system, and the questions of their robotic implementation possibilities. In this essay, we define robot activities and two basic approaches to distance estimation, such as combinatorial planning and sampling-based planning. These two planning strategies are the principles of motion planning. Combinatorial planning is used for finding the path over the continuous configuration space without restoring it to approximations. The most used concept in planning is sampling-based planning, it provides a successful solution in wayfinding path planning, and because of this, it is performed in different robotics fields. Therefore, for distance estimation in IndoorGML maps, we apply combinatorial and sampling-based approaches.
\end{abstract}

Keywords: IndoorGML, Combinatorial Planning, Sampling Based Planning, Probabilistic Roadmap Method, Rapidly-exploring Random Trees.

\section{INTRODUCTION}

These days, navigation and way-finding are becoming essential tasks because people spend much of their time in the indoor setting where way-finding might be difficult. Navigation in a hospital, for instance, where each floor is equally arranged, and the atmosphere is rather monotonous, for inexperienced workers, wayfinding and guidance are difficult tasks. Widespread smartphones will allow us to provide reliable indoor navigation and wayfinding services that are rarely used in indoor environments such as hospitals, airports, shopping centres, etc. For the indoor application of these devices, finding the shortest path to the destination and avoiding the barriers are necessary. Way-finding requires a comprehensive environment definition and a distance estimation function in the static empty indoor environment. Different formats are used to model indoor environments. To model their layout, fixed, and infrastructure, architects, use various models of the same building. These models are generally referred to as the Model of Construction Details. Besides, for indoor navigation purposes, floor plans are preferred and generally accepted. IndoorGML is a new standard used for navigation purposes to model indoor environments. Since IndoorGML only describes the building's structure, to provide way-finding services, a distance estimation feature should be found. Behaviour-based robotics is introduced for situated robots, enabling robots to adjust for the dynamics of real-world environments based on animal behaviour and also providing robots more computational capacity and expressivity. The behaviours are patterns of the activity of the robot that emerges from an external spectator's contact with the robot and its environment. According to a programmer, behaviours are the control modules that cluster constraint collections to achieve and sustain a goal. Each behaviour gathers inputs from sensors or/and from other device behaviours and gives outputs to other behaviours or the actuators of the robot. The behaviour-based controller system is, therefore, a structured network of interacting behaviours with no centralized world representation or control emphasis. In contrast, individual behaviours and behaviour networks maintain some state data and models. For example, we can characterize the dog's ethological robot behaviour such as missing, exploring and fear states, which reflect the conditions in the world. Miss state determines the level of stress of a dog when it is separate from the owner or caregiver, anxiety state explains the level of stress from the conditions of the elderly, and the level of enthusiasm to see in the room is displayed by exploring the state. A suitable robot behaviour is selected from the behavioural set, and ethological awareness of the social behaviour of dogs explains the selection rule and behavioural group, for example, there are several primary dog behaviours, such as dog explores the room and dog moves to the door that dogs display according to the situation. The dog investigates the actions of the room, which defines a dog investigation operation in which the dog watches around in a strange setting, and precisely after a dog enters the room, this form of behaviour can be determined. The dog moves towards the action of the door suggests a typical dog occurrence in which his owner or caregiver is missing from the dog and moves towards the door and stands/sits in front of the door. Different ideas are estimated to contribute to animal intelligence and adaptability such as concurrent activation, hierarchical organizations of behaviour, and coordination of motivational tendencies and individual behaviour excitation and inhibition via thresholds.

Distance approximation is vital for way-finding. Although IndoorGML defines the structure of the building, it does not contain functions for distance 
estimation. The IndoorGML model may contain distance between the objects, but it has to be set manually, which is time-consuming. Hence there is a need for an automatic distance estimation method for IndoorGML. IndoorGML is an Open Geospatial Consortium (OGC)[1] XML-based specification implemented for Indoor Environments. IndoorGML provides an important source of building documentation, it also gives users a context for locationbased applications running and can be of benefit to computer systems where an understanding of the environment is required, IndoorGML purpose is to reflect and facilitate the exchange of geoinformation needed to construct an indoor navigation system. Some facts about indoor spaces are defined in the OGC standard, such as navigation constraints, subdivisions of space, geometric and semantic properties of spaces, and logical and metric navigation networks. IndoorGML combines additional features such as a geometric graph for navigation and a multilayer space model of indoor spaces that apply to indoor navigation with $3 \mathrm{D}$ specifications such as CityGML. IndoorGML uses the Geography Markup language (GML)[2] to encode geo-information and offers a context for the indoor navigation system to suggest a description of the indoor space.

\section{IndoorGML}

Indoor spaces are those spaces within one or multiple buildings which consist of architectural components such as rooms, doors, stairs, corridors, etc. IndoorGML can be defined in two parts, core data model and data navigation model. The core data model is used to describe the topological connectivity, geometry, and different contexts of indoor space. The data navigation model provides semantics for the navigation process (Lee et al [3]) and establishes a methodology to classify spaces and their indoor characteristics. Indoor spatial information is described into two categories. The first category describes the management of building components and indoor facilities, which are used to construct building components such as rooms and walls. The second category is the usage of indoor space, it is defined as usage and localization features in indoor space and represent space components such as rooms, corridors, and doors. Some basic concepts of IndoorGML are Cellular space model, Cell geometry, Topology between cells, and Cell semantics, multi-layered space model, and the last one is the Modular structure of IndoorGML. The IndoorGML uses the concepts of primal and dual space and automatic derivation of dual space that is part of IndoorGML (Diakite et al. [4]). The space subdivision is defined into two significant features such as primal space and dual space. Primal Space describes the control of the indoor space that influences the representation of indoor cells and the dual space describes the information of the norm to support the automation of the space subdivision process. The classification of IndoorGML can be represented in 2D, 3D, and the link between indoor and outdoor. (Kim and Lee, [5]) have proposed an approach called semi-automatic, to create IndoorGML data from images, and within the same direction, Mirvahabi and Abbaspour have introduced an automated method to extract IndoorGML data from OpenStreetMap [6]. The purpose of IndoorGML is to follow the requirements for indoor navigation by supporting the common framework for determining the spatial models for indoor navigation.
The extensible markup language design for IndoorGML core module consists of four basic types such as CellSpace, CellSpaceBoundary, State and Transition. The First two CellSpace and CellSpaceBoundary are the key units of the primal space of IndoorGML, while as the last two State and Transition belongs to the dual space of IndoorGML.

Primal Space describes the control of the indoor space that influences the representation of indoor cells and the main components of primal space of IndoorGML are CellSpace and CellSpaceBoundary [7]. CellSpace is used to determine the main types of the cellular indoor spatial model, such as room, corridor, and hall, and it also includes a GML identifier (an identifier which is assigned to an object by the maintaining authority which is used in references to the object), this identifier is usually unique either globally or inside an application domain with its attributes. The cell geometry for primal space is described by the International Organization for Standardization (ISO 19107 [8]), which can be either surface or solid depending upon the dimensionality of space object either 2D or 3D. The CellSpaceBoundary describes the geometric edges of a CellSpace object, and its geometry may be either surface or curve depending on the dimensionality. Different options are employed to define the geometry of CellSpaceBoundary, the first one is to include only topological relationships between cells. The second is to present the geometry within IndoorGML data by geometric types defined in ISO 19107. The last one requires external references to the object in another dataset that has geometric data. The location class of Primal Space can be described based on some different concepts such as construction, functional area, and security, for building and walking and driving for the user. Each specific location area can be formulated in a space layer, i.e., a multi-layered space model, in which spaces from different types of layers may overlap and no overlapping is allowed between the cells in the same space layer.

Dual space describes the information of the norm to support the automation of the space subdivision process and this dual space is derived from the primal space and used to describe the node relation graph (NRG). Node relation graph describes the topological relationship between indoor spatial environments such as adjacency and connection (Lee, 2004 [9]). The basic thoughts which are employed in dual space are state and transition [7]. They describe the feature types of dual space equivalent to cellspace and cellspace boundary that is already defined in primal space in terms of the connectedness of topologies. In dual space nodes represent cellspaces and edges represent transitions. In the Dual Space graph, the dashed line represents the non-navigable links that are the adjacency of graph e.g. walls, obstacles, etc, and the bold line represents navigable links that is the connectivity of graph e.g. doors, rooms, corridors, etc. To navigate from one room to another and find the distance between them, just go through the bold lines because these lines show the connectedness of rooms, and then to determine the shortest distance Dijkstra algorithm is applied if the distances or costs are known.

\section{ROBOT BEHAVIORS}

Behavior-Based Robotics is a behavior what an external observer sees a robot doing. Robots are programmed to display desired behavior. For example behavior of a robot, 
one behavior task can focus on traversing a path from start to goal state, while the other one focus on avoiding obstacle. However there are some fundamental ways to describe robot behavior such as Deliberative, Reactive, and Hybrid, Behavior-Based Control [10].

Deliberative control method in this the robot is going to uses all its internally stored knowledge and available sensory information to reason regarding what actions to be taken next. Robot decision making is described as the process in which the robot is getting information regarding the environment with the robot's sensors, preparing it as essential in the form to build decisions about how to act, and performing activities in the environment. Reasoning in deliberative systems occurs usually in the form of planning, needing an exploration of possible state-action sequences and their consequences. Planning needs the occurrence of an internal and symbolic representation of the world that supports the robot to watch forward into the future and predict the results of possible actions in many states to generate plans. Meanwhile, the internal model needs to be maintained accurate and up to date, if there is enough time to generate a plan and the world model is accurate, this strategy enables the robot to behave strategically, choosing the most suitable way of action for a given situation.

Reactive control method for tightly coupling sensory inputs and effector outputs, usually including no intervening reasoning to allow the robot to respond very immediately to developing and unstructured environments. Reactive control is encouraged by the biological knowledge of stimulus-response, there is no need for acquisition or maintenance of world models. Reactive control does not react on the kinds of complex reasoning rules employed in deliberative control rather it uses a rule-based method that involves a minimum quantity of calculation. Reactive systems perform fast real-time responses by mapping the robot's controller in a group of the preprogrammed, simultaneous conditionaction rules with the minimum inner state that produces a well suited reactive control for dynamic and unstructured worlds. Reactive systems are able to react suitably for quickly changing environments with the presence of minimum quantity of calculation [11].

Hybrid control method merges the advantages of both reactive and deliberative control: such as the real-time reply of reactivity and the rationality and optimality of deliberation. As a conclusion, the hybrid control method includes a pair of different components such as the reactive/simultaneous condition-action rules and the deliberative decision making, which need to communicate in order to construct an understandable output [12]. The method is challenging because the reactive component is based on the robot's quick requirements, such as moving towards the target while avoiding obstacles, and therefore operates on a very fast time scale and applies direct external sensory data and signals, while the deliberative component applies extremely abstracted, symbolic, internal descriptions of the world, and perform functions on them upon a longer time scale such as to perform global path planning or plan for high-level decisionmaking. The reactive system needs to override the deliberative system if any unexpected and immediate challenge existing in the world and the deliberative system should inform the reactive system to guide the robot to more efficient and optimal trajectories and goals.
Behavior-based control system operates on a set of distributed, interacting modules known as behaviors that collectively accomplish the desired system-level behavior. The behaviors are patterns of the robot's action developing from communications between the robot and its environment in an outside spectator. Accordingly to a programmer, behaviors are the control modules that cluster collections of constraints in order to obtain and maintain a goal. Each behavior collects inputs from sensors or/and from other behaviors in the system and gives outputs to other behaviors or to the robot's actuators. Therefor behavior-based controller system is a structured network of interacting behaviors with no centralized world representation or focus of control while individual behaviors and networks of behaviors keep any state information and models. Well-designed behavior-based systems holds the advantage of the dynamics of communication within the behaviors themselves, and between the behaviors and the environment [13]. Unlike reactive control system that applies the collections of reactive rules with few if any state and no representation while as the behavior-based control system applies sets of behaviors which have state that can be employed to build representations, thereby enabling reasoning, planning, and learning.

Wall Following behavior [14] example for robot behavior navigation. In this Robot can follow the wall, therefore allowing this to walk along the boundaries of the testing environment. The basic procedure for wall following procedure is shown in figure 1 . In this, the robot uses ultrasonic sensors to detect the left and right side distances ( $\mathrm{dr}$ and $\mathrm{dl})$. The sensing system determines the side distances of the robot, later on it judges which side (left or right) is nearby to the robot and selects the distance to the nearest side as the baseline, allowing the robot to move along the nearest wall. After moving to the nearest wall the robot estimates the current location against that in the previous (far away from or closer to the sidewall). If the distance of the previous is lesser than of current, then it implies that the robot moved farther from the wall and then the robot needs to turn toward the wall. If the distance of the current is shorter than previous then it implies the robot has moved closer to the wall and the robot must turn away from the wall now. de is the difference between the wall distances in the two cycles. Fuzzy logic controller takes two inputs de and vc. vc is the relatively fast velocity which means that the robot has obtained significant speed by moving away from or closer to the wall. $\theta e$ is the output of the fuzzy controller which allows the robot to turn toward the wall and return to correct the wall-following behavior.

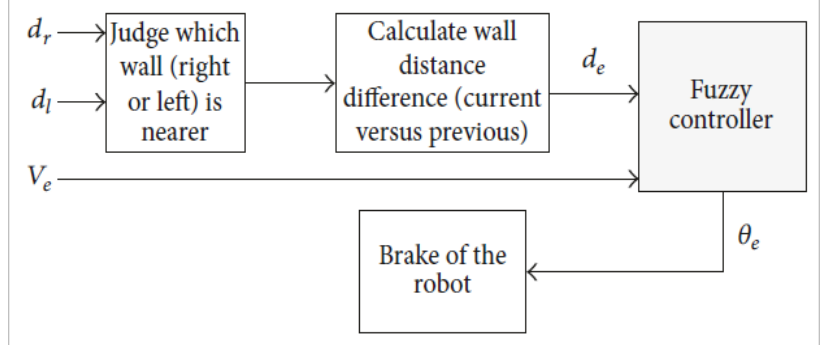

Figure 1. Wall following behavior procedure 


\section{COMBINATORIAL PLANNING}

Combinatorial planning is an approach of motion planning algorithm [15]. The motion planning algorithm can be expressed such as a robot is given with its initial state, final state, and geometric description of the robot and world. Then determine a path or sequence of valid configurations that move the robot gradually from an initial to the final state without hitting any obstacle. The motion planning algorithm approach is also referred to as piano mover's problem in which a piano is presented to move from one room to another without hitting an obstacle. Translation and rotation are the main ideas in robot motion planning that is required to move a robot. The C-space (configuration space) is the key idea in motion planning. It is the space of all possible (valid) configurations and can also be described as a topological manifold (is a topological space in which for any two distinct points there exists a neighborhood of each of them but which is disjoint from the neighborhood of the other also called euclidean hausdorff space). There are two basic approaches to represent $\mathrm{C}$-space [16] such as combinatorial and sampling-based planning. Combinatorial planning is finding the path over the continuous $\mathrm{C}$ - space without resorting to approximations. Different methods are defined in combinatorial planning such as visibility graphs, voronoi diagrams, and exact cell decomposition.

Visibility graph [17] is defined as the graph of intervisible locations, usually for a set of points and obstacles in the Euclidean plane. In this graph, each node represents a point location (stop area), and each edge shows a visible connection, shown in figure 2(a). The basic idea is to draw a straight line from start $(\mathrm{qI})$ and goal (qG) to all visible vertices, and then again draw a straight line from one vertex to all visible vertices. After connecting these vertices the shortest path is shown as in figure 2(b).

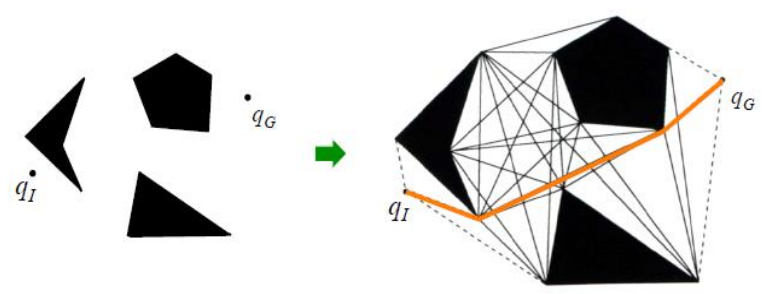

Figure (a).

Figure 2(b)

Figure 2. Visibility Graph

Voronoi diagram [18, 19] division of a plane into regions based on distance to points in a specific subset of the plane. In this set of points that are closest to two or more obstacles boundaries are set to equal distance, the basic idea in this is to maximize the clearance between the points and obstacles as shown in figure 3. And then calculate the shortest path (by using algorithms) from start to goal. The path yielded will be equally from each obstacles. Three types of nodes exist in the voronoi diagram such as intersection nodes, terminal nodes, and pseudo-nodes. An intersection node is formed where three or more arcs of the voronoi diagram intersect. A terminal node compares to a dead end of a voronoi diagram arc and the terminal nodes appear during curved vertices are present. Pseudo-node also called a source or a goal node, these are the artificial nodes inserted in the graph near to the source and goal states individually and these nodes also represent entry and exit points.

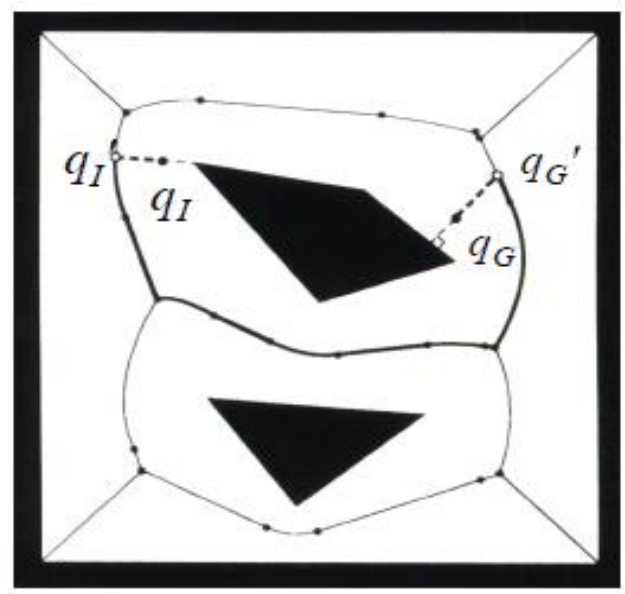

Figure 3. Voronoi diagram

Exact cell decomposition [16] is defined as the division of free space in C-space into cells, shown in figure 4 . The basic method is decompose the free space with vertical lines from vertices without crossing a forbidden space (obstacle space). Mark the center to each of these vertical vertices and trapezoid a graph node and add centre point between the two vertical lines. Find shortest path in this graph with a graph searching algorithms such as Dijkstra's algorithm [20] etc.
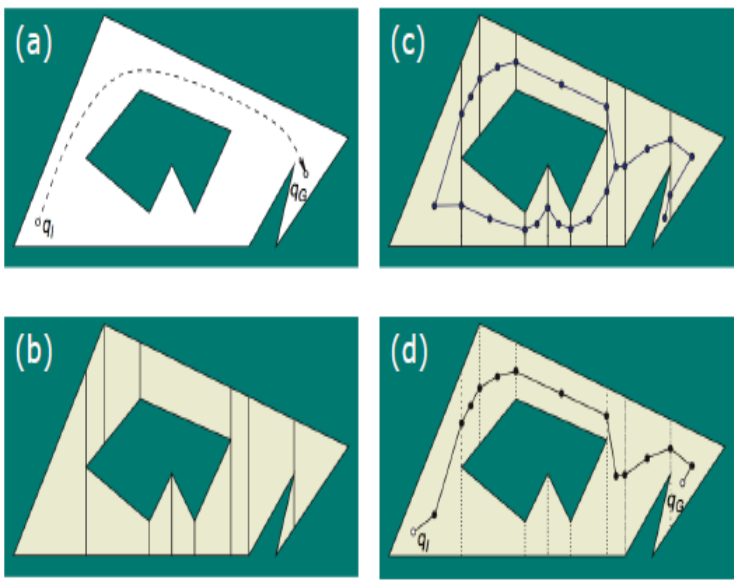

Figure 4. Exact cell decomposition

\section{SAMPLING BASED PLANNING}

Sample Based Planning (SBP) is the fact that planning occurs by sampling the configuration space (C-space [21]). The responsiveness of the sampling-based planning 
approach is to try to catch the connectivity of the C-space by sampling it. Sampling-based planning gives a feasible solution because it is treated as a black box that means it returns a collision-free path once information about the start and goal configurations is provided, as shown in figure 5. Probabilistic Roadmap Method (PRM) and Rapidly-exploring Random Trees (RRT) are the two basic algorithms that are used in sampling-based planning. In SBP figure several terms are used such as Sampling, Metric, and Nearest Neighbor, Local planning, etc. Sampling means to select a configuration randomly and add it to the tree or roadmap. The selected samples can be present either in the free, or obstacle configuration space. In metric, given two configurations qa and $\mathrm{qb}$, this procedure returns a value, or cost, that signees the effort required to reach qb from qa. Nearest-Neighbor (NN) is a searching algorithm that returns the closest node(s) to the new sample. Select Parent says to select an existing node to connect to the newly sampled node and the existing node is considered a parent. Given two configurations qa and $\mathrm{qb}$ then Local planning attempts to establish a connection between these configurations. Collision checking (CC) is mostly a Boolean function that returns true (success), or false (failure) when connecting two configurations. A connection is successful if it does not intersect Cobs.

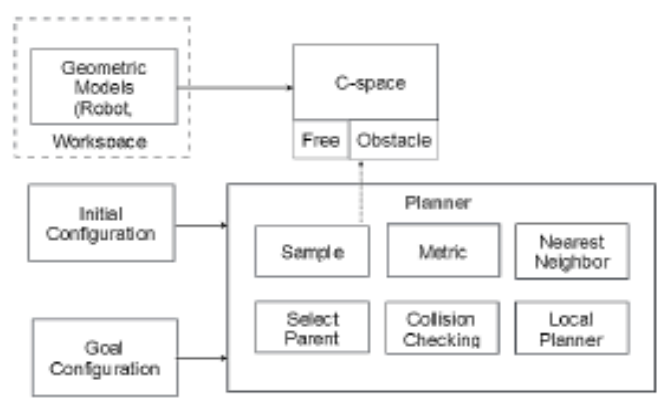

Figure 5. General procedure for sampling based planner

Probabilistic Roadmap Method (PRM) is applied to resolve the problem of deciding a path from a start to goal configuration of the robot while avoiding collisions. The basic idea of PRM is to take random samples from the configuration space of the robot [22], testing them for whether they are in the free space if so declare them as vertices, and use a local planner to try to connect them to other nearby vertices in configurations space. The two basic keywords that are used in PRM are Learning and query phase. In the Learning phase the first idea is to find the random sample of free configuration space and try to connect pair of nearby vertices of free $\mathrm{C}$-space with the local planner if the possible valid path is found then add the edges to the graph. In the Query phase find the local connection of graph from start to goal positions, then use A* searching algorithms [23] to find the path. PRM is also called a multi-query planner. In PRM firstly it is required to construct the roadmap [24] in the learning phase and after roadmap construction we can describe the basic steps of this algorithms.

- By sampling method select a random node q-random from the configuration space.

- If q-random is found in Cobs (obstacles) then q-random is discarded otherwise add q-random to the roadmap.

- With the help of nearest neighbor algorithm find all the nearest nodes that are within the range of q-random using nearest-neighbor algorithm.

- Try to connect all these neighboring nodes to q-random using the local planner method.

- Checking for collision [25], if collision is found then disconnect the colliding paths.

- Repeat the process until the certain number of nodes are sampled.

Rapidly-exploring Random Trees (RRT) in this the basic intention is to select the random configuration from configuration space [26] if the selected random configuration is present in free space then try to connect the nearest vertex in the tree. The tree is growing incrementally from start to goal configuration. RRT also is referred to as a single-query planner and mostly used for single queries because it is faster than PRM that is RRT does not need to construct the roadmap i.e, learning phase. The following steps describe the working procedure of the RRT algorithm.

- Initialize with q-start.

- Select a random node q-random from the C-space using the sampling method.

- Discard the random node (q-random-discard) if it is found in obstacle space Cobs.

- Use Nearest Neighbor search q-near which is returned to see nearby configuration according to the metric.

- Connect the q-random and q-near using the local planner method. The local planner may recover q-new*qrandom, that may not be accessible (reachable). If it is not reachable then it is discarded.

- Check for the collision to ensure that the path from qnear and q-new is collision free or not. If the path is collision free then q-new is added to the tree.

- Terminates the search (q-near) when q-new= q-goal.

\section{CONCLUSION}

IndoorGML is an XML-based standard of Open Geospatial Consortium introduced for Indoor Environments and it presents an important source of documentation of buildings, it also gives context to users operating location-based applications. IndoorGML describes the indoor environment and IndoorGML defines some basic concepts such as Cellular space model and Cell Geometry, Topology between cells and Cell semantics, multi-layered space model, and the core part Modular structure of IndoorGML. The core part of IndoorGML describes the basic components of the IndoorGML data model. It includes the schema definitions of basic classes for cell geometry, topology between cells, and multi-layered space model, and it also provides the semantic extension model for indoor 
navigation. The measurement of indoor distances is accomplished by horizontal and vertical distances and multi-modal transportation. The two main components that are described in IndorGML is Primal and Dual Space. The primal spaces are used to define the main types of the cellular indoor spatial model, such as room, corridor, and hall, and it also defines the edges of the geometric object, and its geometry maybe both surface or curve depending on the dimensionality. The dual spaces are derived from primal spaces, but in dual space, nodes represent cell-spaces, and edges represent transitions.

Choosing a suitable control behavior and designing a structure in it is best described by the situatedness properties of the problem, the nature of the task, the level of efficiency or optimal needed, and the capabilities of the robot, both in terms of computation, hardware, and world modeling. For example, reactive control is the most suitable choice for circumstances requiring an instant reply but such activity of reaction occurs at the price of being myopic not seeing into the past or the future. Deliberative systems are the only opportunity for domains that require a vast understanding of strategy and optimization, and in-service exploration and planning. Hybrid systems are suitably for environments and tasks where planning and internal designs are needed, and also the real-time requirements are few or adequately independent of the higher-level reasoning. Behaviorbased systems, in contrast, are completely fit for environments including significant dynamic changes, where quick reply and adaptivity are significant, but the capability to do some watching progressing and avoid past mistakes is required.

Initial achievement in planning is to develop deterministic planning techniques. In motion planning, a robot is given with its start state, goal state, and geometric description of robot and world, and then find a path or sequence of valid configurations that move the robot gradually from start to goal while never touching any obstacle. Translation and rotation are the main focus in robot motion planning that is needed to move the robot. Combinatorial planning is used to find the path over the continuous configuration space without resorting to approximations and in this method, we define some concepts such as visibility graph, Voronoi diagram, and exact cell decomposition. After determining these methods it is required to try to connect the connectivity of start to goal state to find the valid optimal path using some graph searching algorithms such as Dijkstra. Simple based planning produces essentially in the configuration space (C-space). Configuration space is a space of all possible transformations such as free space and obstacle space. Combinatorial planning algorithms could be applied in a static environment where the obstacles are stationary while Sample-based planning methods are more complex and can be used dynamically changing environment. Because the layout of the building never changes, the usage of Combinatorial Planning algorithms is suggested. Visibility Graph yields a path which approaches the obstacles as close as possible. Hence the Visibility Graph method would give a good approximation of natural movement. The result of the Voronoi Diagram based method is quite the opposite of the previous one because it creates a route that is as far the obstacles as possible and this method seems to be quite useful for autonomous devices.

\section{REFERENCES}

[1] Markus Lupp. Open geospatial consortium. In Encyclopedia of GIS, pages 815-815. Springer, 2008.

[2] Portele Clemens. Geography markup language (gml) encoding standard, 2007.

[3] Abdullah Alattas, Sisi Zlatanova, Peter van Oosterom, and KiJoune Li. Improved and more complete conceptual model for the revision of indoorgml (short paper). In LIPIcs-Leibniz International Proceedings in Informatics, volume 114. Schloss Dagstuhl-Leibniz-Zentrum fuer Informatik, 2018.

[4] Abdoulaye A Diakite' Sisi Zlatanov, and Ki-Joune Li. About the subdivision of indoor spaces in indoorgml. ISPRS Annals of Photogrammetry, Remote Sensing \& Spatial Information Sciences, 4, 2017

[5] M Kim and J Lee. Developing a method to generate indoorgml data from the omni-directional image. The International Archives of Photogrammetry, Remote Sensing and Spatial Information Sciences, 40:17, 2015.

[6] SS Mirvahabi and RA Abbaspour. Automatic extraction of indoorgml core model from openstreetmap. The International Archives of Photogrammetry, Remote Sensing and Spatial Information Sciences, 40(1):459, 2015

[7] Kang, H.K. and Li, K.J., 2017. A standard indoor spatial data model-OGC IndoorGML and implementation approaches. ISPRS International Journal of Geo-Information, 6(4), p.116.

[8] TC ISO. 211. ISO 19107 geographic information-spatial schema, 2003.

[9] Lee, J. 2004. A Spatial Access Oriented Implementation of a Topological Data Model for 3D Urban Entities, GeoInformatica 8(3), 235-262.

[10] Siciliano, Bruno, and Oussama Khatib, eds. Springer handbook of robotics. Springer, 2016.

[11] Vadakkepat, Prahlad, et al. "Fuzzy behavior-based control of mobile robots." IEEE Transactions on Fuzzy Systems 12.4 (2004): 559-565.

[12] J.H. Connell: SSS: A hybrid architecture applied to robot navigation, Proc. IEEE Int. Conf. Robotics Autom. (1992) pp. 2719-2724

[13] Towle, Bradford A., and Monica Nicolescu. "Real-world implementation of an Auction Behavior-Based Robotic Architecture (ABBRA)." 2012 IEEE International Conference on Technologies for Practical Robot Applications (TePRA). IEEE, 2012.

[14] Chen, Ching-Han, et al. "Fuzzy logic controller design for intelligent robots." Mathematical Problems in Engineering 2017 (2017).

[15] LaValle, Steven M. Planning algorithms. Cambridge university press, 2006.

[16] Burgard, Wolfram, et al. "Introduction to mobile robotics." University of Freiburg (2011).

[17] Asano, T., Asano, T., Guibas, L., Hershberger, J., \& Imai, H Visibility-polygon search and euclidean shortest paths. The 26th Annual Symposium is Foundations of Computer Science (SFCS 1985) (pp. 155-164). IEEE.

[18] Canny, John. "A Voronoi method for the piano-movers problem." Proceedings. 1985 IEEE International Conference on Robotics and Automation. Vol. 2, IEEE, 1985

[19] Takahashi, O., \& Schilling, RJ (1989). Motion planning in a plane using generalized Voronoi diagrams. IEEE Transactions is robotics and automation, 5 (2), 143-150.

[20] Dijkstra, Edsger W. "A note on two problems in connexion with graphs." Numerische mathematik 1.1 (1959): 269-271.

[21] Elbanhawi, Mohamed, and Milan Simic. "Sampling-based robot motion planning: A review." Ieee access 2 (2014): 56-77. 
[22] Amato, Nancy M., and Yan Wu. "A randomized roadmap method for path and manipulation planning." Proceedings of IEEE international conference on robotics and automation. Vol. 1. IEEE, 1996.

[23] Hart, Peter E., Nils J. Nilsson, and Bertram Raphael. "A formal basis for the minimum cost paths." IEEE transactions are Systems Science and Cybernetics 4.2 (1968): 100-107.

[24] L. E. Kavraki, P. Svestka, J. C. Latombe, and M. H. Overmars, "Probabilistic roadmaps for path planning in high-dimensional conguration spaces," IEEE Trans. Robot. Autom., vol. 12, no. 4, pp. 566_580, Aug. 1996.

[25] Sánchez, Gildardo, and Jean-Claude Latombe. "On delaying collision checking in PRM planning: Application to multi-robot coordination." The International Journal of Robotics Research21.1 (2002): 5-26.

[26] M. LaValle, "Rapidly-exploring random trees: A new tool for path planning," Dept. Comput. Sci., Iowa State Univ., Ames, IA, USA, Tech. Rep. TR 98-11, 1998. 


\section{Poojarini Mitra et. al., American Journal of Electronics \& Communication, Vol. I (3), 8-11 \\ Character Segmentation for Handwritten Bangla Words using Image Processing}

Poojarini Mitra, Kaustav Bhattacharjee, Anirban Das, Sayan Kumar Dey, Deepjyoti Chakraborty, Aritra Ghosal, Shadab Akhtar

Computer Science Engineering

University of Engineering and Management

Kolkata, India

poojarini.mitra@uem.edu.in, kaustuv.bhattacharjee@uem.edu.in, anirbandas01011984@gmail.com, sayankumardey908@gmail.com, deepjyoti19982018@gmail.com, aritra.ghosal.96@gmail.com,shadabdsw@gmail.com

\begin{abstract}
Character segmentation has long been a critical area of the OCR process. The higher recognition rates for isolated characters vs. those obtained for words and connected character strings well illustrate this fact. A good part of recent progress in reading unconstrained printed and written text may be ascribed to more insightful handling of segmentation.
\end{abstract}

To take care of variability involved in the writing style of different individuals in this paper we propose a robust scheme to segment unconstrained handwritten Bangla texts into lines, words and characters. For line segmentation, at first, we divide the text into vertical stripes. Stripe width of a document is computed by statistical analysis of the text height in the document. Next we determine the horizontal histogram of these stripes and the relationship of the minimal values of the histograms is used to segment text lines. Based on vertical projection profile lines are segmented into words. Segmentation of characters from handwritten words is very tricky as the characters are seldom vertically separable. Segmentation of cursive handwriting is the challenging step of Optical Character Recognition (OCR).The recognition accuracy will highly depend on the good segmentation. Segmentation of cursive handwriting is very difficult. The segmentation can be done on the basis of zoning, a line segment of text, a word segment from line and character segment from word. This can be done by the use of horizontal, vertical methods. This paper reviews many basic and advanced techniques of handwritten word segmentation.

\section{INTRODUCTION}

Optical character recognition is a program that translates a scanned image of a document into a text document that can be edited. Segmentation of cursive handwriting is very difficult. Character segmentation is an operation to decompose an image into the sub-image of individual symbols. There are mainly three phases of a character recognition system, namely pre- processing, segmentation and recognition. Pre-processing aims to produce data that is easy for the OCR system to work accurately. It reduces noise and distortion, removes skewness and performs skeletonizing of the image, thereby simplifying the processing of the rest of the stages. The next stage is segmenting the document into its sub-components. It separates the different logical parts, like text from graphics, line of a paragraph, and character of a word. Segmentation of unconstrained handwritten text lines is difficult because of inter-line distance variability and base-line skew variability. Components of two consecutive text-lines may be touched or overlapped in unconstrained handwritten text. These overlapping or touching characters complicate the line segmentation task greatly. In Bangla touching or overlapping occurs frequently because of modified characters of upper-zone and lower- zone. Most of the characters in Bangla handwritten words are touching and segmentation of touching characters is the main bottleneck in the handwritten recognition system. Many techniques have been proposed on touching character segmentation. One class of approaches uses contour features of the component for segmentation. Some researchers use profile features for touching character segmentation. Thinning based methods are also reported for touching characters segmentation. Combined features based methods are also used for the touching string segmentation. Although many methods on handwritten line, word and character segmentation have been published in the literature for Roman, Chinese, Japanese and Arabic scripts only one report is available on character segmentation from Bangla handwritten isolated words. They used recursive contour following technique for character segmentation from a word. In this paper we propose a robust scheme to segment handwritten texts of Bangla script into lines, words and characters. Bangla is the second-most popular language in the Indian subcontinent and fifth-most popular language in the world. For line segmentation we divide the text into vertical stripes and determine horizontal histogram projections of these stripes. The relationship of the minimal values of the histograms is used to segment text lines. Based on vertical projection profiles, lines are segmented into words. Segmentation of characters from handwritten words is difficult as the characters are mostly connected in a word. For character segmentation we first detect isolated and touching characters in a word. 


\section{PROPERTIES OF BANGLA SCRIPT}

The alphabet of the modern Bangla script consists of 11 vowels and 39 consonants. These characters may be called basic characters. The basic characters of Bangla script are shown in Fig.1. Writing style in the script is from left to right. The concept of upper/lower case is absent in Bangla script.

\begin{tabular}{|c|c|c|c|c|c|c|c|c|c|c|}
\hline & আ & ই & $\bar{x}$ & উ & ঊ & $\psi$ & @ & ঐ & 3 & () \\
\hline & ₹ & গ & घ & is & চ & ছ & G & ঝ & $\Omega$ & $\vec{b}$ \\
\hline & 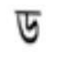 & ঢ & ণ & ত & ข & $\overline{4}$ & 8 & ন & भ & \\
\hline & ভ & ম & য & র & ल & শ & ষ & স & ₹ & \\
\hline & য় & $\rho$ & $i$ & : & * & & & & & \\
\hline
\end{tabular}

Fig.1. Basic characters of Bangla alphabet. (First 11 are vowels and rest are consonants)

From Fig.1.it is noted that most of the characters have a horizontal line at the upper part. When two or more characters sit side by side to form a word, these horizontal lines touch and generate a long line called head-line. We use these head-line characteristics for isolated and connected character identification and character segmentation. From a statistical analysis we notice that the probability that a Bangla word will have at least one character with head-line is 0.994. Hence, the use of headline based features is justified for the purpose. In Bangla script a vowel following a consonant takes a modified shape, which depending on the vowel, is placed at the left, right (or both) or bottom of the consonant. These are called modified characters. A consonant or vowel following a consonant sometimes takes a compound orthographic shape which we call as compound character. Compound characters can be combinations of consonant and consonant, as well as consonant and vowel. A Bangla text line can be partitioned into three zones. The upper-zone denotes the portion above the head-line, the middle zone covers the portion between head-line and base-line, the lower-zone is the portion below base-line.

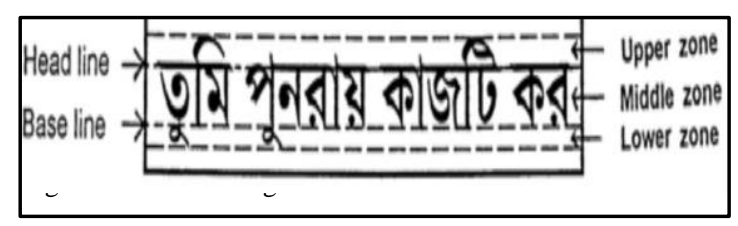

III. LINE, WORD AND CHARACTER SEGMENTATION

Segmentation processes, including following processes:

\section{A. Line segmentation}

Line segmentation is the process in which from the image, we extract only lines or differentiate the lines. Horizontal projection of a document image is most commonly used to extract the lines from the document. The horizontal projection will have separated peaks and valleys for the lines that are well separated and are not tiled, which serve as the separators of the text lines. These valleys are easily detected and used to determine the location of boundaries between the lines. Word segmentation is the process in which from the line segmentation, we extract only words. As we know that there is a distance between one words another word, this concept is used for word segmentation.

\section{B. Word segmentation}

Word segmentation is a process of dividing a string into its component words. Word splitting is the process of parsing concatenated text to infer where word breaks exist. By using a vertical projection profile, one can get column sums. By looking for minima in the horizontal projection profile of the page, we can separate the lines and then separate words by looking at minima in the vertical projection profile of a single line. By using the valleys in the vertical projection of line image, one can extract words from a line and also extract individual characters from the word. The global horizontal projection method computes the sum of all black pixels on every row and constructs a corresponding histogram. Based on the peak/valley points of the histogram individual lines are segmented. This method has some drawbacks: (a) it will not work on skewed texts (b) it will not work on overlapping situations (c) some diacritical points in Bangla can generate false separating lines. To take care of above drawbacks we use a modified piecewise projection method suitable for Bangla script. Here we assume that a document page is in portrait mode. In this method we divide the text into vertical stripes of width $\mathrm{W}$. Width of the last tripe may differ fromW. If the text width is $\mathrm{Z}$ and the number of stripe is $\mathrm{N}$ then the width of the last stripe is $\left[\mathrm{Z}-\mathrm{W}^{*}(\mathrm{~N}-1)\right]$. Computation of $\mathrm{W}$ is discussed later. Next we compute Piecewise Separating Lines (PSL) from each of these stripes. We compute the row wise sum of all black pixels of a stripe. The row where this sum is zero is a PSL. We may get a few consecutive rows where the sum of all black pixels is zero. Then the first row of such consecutive rows is the PSL. The PSLs In character segmentation, we extract only characters from word. Character segmentation is a difficult step of OCR systems as it extracts meaningful regions for analysis. This step decomposes the images into classifiable units called characters. A poor segmentation process leads to incorrect recognition or rejection segmentation process carried out only after the preprocessing of the image of different stripes of a text are shown by black horizontal lines. All these PSLs may not be useful for line segmentation. We choose some potential PSLs among these. We compute the normal distances between two consecutive PSLs in a stripe. So if 
there are $n$ PSLs in a stripe we get $n-1$ distances. This is done for all stripes. We compute the statistical mode (MPSL) of such distances. If the distance between any two consecutive PSLs of a stripe is less than MPSL we remove the upper PSL of these two PSLs. PSLs obtained after this removal are the potential PSLs. The potential PSLs of Fig.4(a) are shown in Fig.4(b). We note the left and right coordinates of each PSL for future use. By proper joining of these potential PSLs we get individual text lines. It may be noted that sometimes because of overlapping or touching of one component of the upper line with a component of the lower line, we may not get PSLs in some regions. We will take care of these situations during PSLs joining.

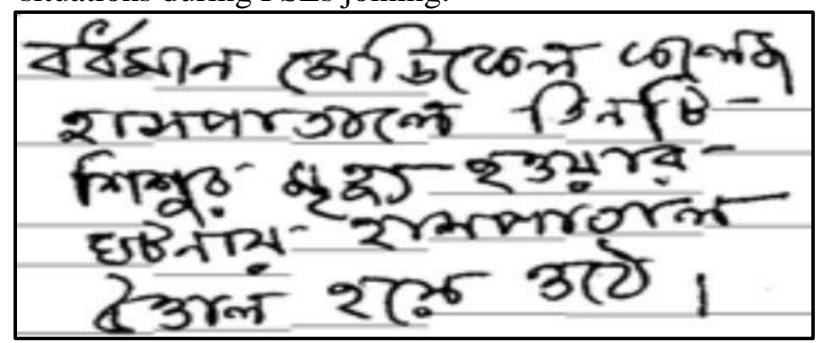

Fig.3: PSLs and potential PSLs are shown in (a) and (b)respectively, for a Bangla handwritten text

\section{Character Segmentation}

In character segmentation, we extract only characters from words. Character segmentation is a difficult step of OCR systems as it extracts meaningful regions for analysis. This step decomposes the images into classifiable units called characters. A poor segmentation process leads to incorrect recognition or rejection segmentation process carried out only after the preprocessing of the image.

\section{SKEW DETECTION AND CORRECTION}

Prior to the segmentation it is necessary to preprocess all word images. Initially the images are in a new variable and are used to create the histogram. Each element of this variable represents the number of black pixels of the main word image. Black pixels are then plotted in an image file to get the histogram grayscale format. The gray scale is median filtered and then using the algorithm is used to binarize the images. Document skew is a distortion that often occurs during document scanning and copying. This mainly concerns the orientation of text lines and with no skew the lines are horizontal or vertical, depending on the language. This effect visually appears as a slope of the text lines with respect to the $\mathrm{X}$ axis. Document skew is an unavoidable effect because of the complex structure of handwritten words and the copying and scanning process especially when digitizing the huge amount of documents.

\section{CHARACTER SEGMENTATION}

After word segmentation and skew correction we are getting each word as different images. Now on each word we are segmenting each character for recognition. In the beginning inputting each word in grayscale, it is noticed that some pixels in the images are gray. To eliminate those pixels, the image is converted into black and white.

\section{Fig.4: Original Word}

The output is then saved as an image variable.

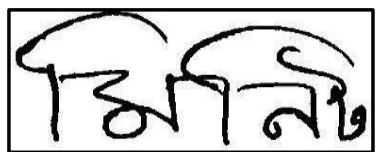

On that image variable, the black pixels are counted for each row and it is saved in a new variable. This new variable is used to create the histogram. Each element of this variable represents the number of black pixels of the main word image. Black pixels are then plotted in an image file to get the histogram.

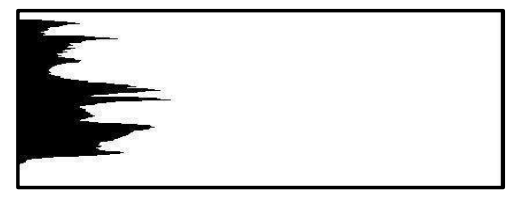

Fig.5: Histogram of Original Word

In the histogram it is noticed, in the "Matra" region the occurrence of black pixels increased drastically if compared to the change in occurrence of black pixels for the rest of the image. So we calculated the highest change in occurrence to determine the "Matra" region of the image. Now from that very point (region) we segregated the upper zone and middle zone of the word and saved the segregated images into files. The upper zone is saved as "upper" and the middle zone is saved as "mid".
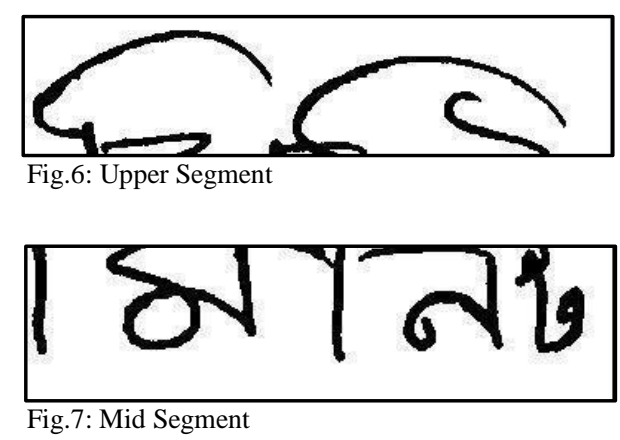

As we already got the middle zone now it's all about segmenting the middle zone and upper zone for character segmentation. To do so we copied the image "mid" into a new variable. In most of the cases after 
segregation from the "Matra" region the "Matra" is eliminated. We noticed on multiple words that there is a minimum of 4 pixel gap in between characters. So we drew some black lines on the image "mid" in a way so that if there is any black pixel occurrence on those drawn lines, those pixels will become white.

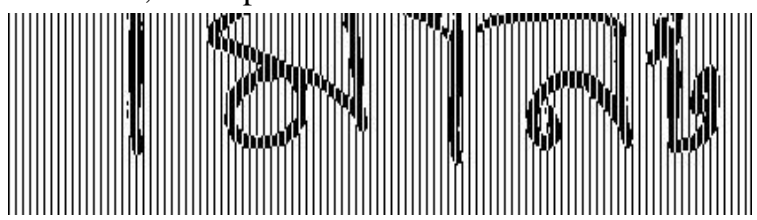

Fig.8: Modified Mid Segment

Now in this new image only those lines will black, which does not go through any character. The column index of those black lines is saved in a list variable. There can be multiple black lines one after the other. To eliminate those extra black lines we calculated, if the difference of two corresponding elements of the list is 4 , the first element is removed. In this way we got the segmentation points. Using these segmentation points the "mid" image is segmented and each segmented image is saved in image files. The image files are numbered depending upon the segregation number.

\section{CONCLUSION}

In this paper we have proposed a scheme for Segmentation of unconstrained handwritten words into different zones(upper and lower) and characters. There are many difficulties in respect to segmenting handwritten words of Bangla script. Most of these difficulties can be encountered due to the properties of Bangla script. In this paper we propose a simple method to segment unconstrained Bangla words. We achieved some success rate in the proposed system. The word and character segmentation is a kind of a procedure which will be of great help in the field of Bangla handwritten word recognition. In the future this segmentation can be helpful for developing an automated Bangla handwriting recognizer and analyzer. This can be also helpful in future for graph logical analysis of handwritten documents.
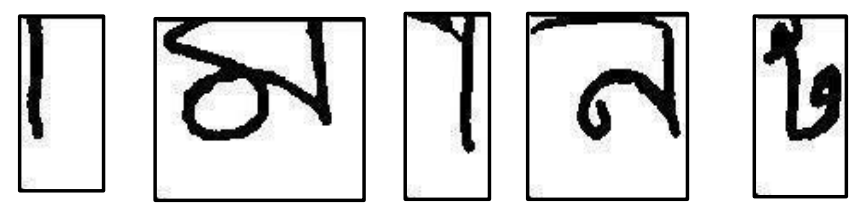

Fig.9: Character Segmentation of Mid Segment
[2] T. K. Bhowmik, A. Roy and U. Roy, "Character Segmentation for Handwritten Bangla Words using Artificial Neural Network"

[3] B.Chaudhari and U.Pal, "Skew angle detection of digitized Indian Script documents".

[4] A.Bishnu and B.B.Chaudhari "Segmentation of Bangla handwritten text into characters by recursive contour following".

[5] R.G. Casey, E. Lecolinet, “A survey of methods and strategies in Character segmentation.

[6] K. Kurino, Y. Nakano, H. Fujisawa, "The book of segmentation methods for character recognition from segmentation to document structure analysis. 


\section{Android Controlled Home Automation}

\author{
Pankaj Kapoor \\ department of Computer Science \\ University of Engineering \& \\ Management, Kolkata \\ Kolkata, India \\ pankajkapoor580@gmail.com \\ Prof. Dr. Sudipta Basu Pal \\ Assistant Professor, Department of \\ Computer Science \\ University of Engineering \& \\ Mangement, Kolkata \\ Kolkata, India \\ sudipta.basu@uem.edu.in
}

\author{
Suryadipta Sarkar \\ department of Computer Science \& \\ Technology \\ University of Engineering \& \\ Managtement, Kolkata \\ Kolkata, India \\ suryadipta.sarkar00@gmail.com \\ Kunal Mahato \\ department of Computer Science \\ University of Engineering \& \\ Management, Kolkata \\ Kolkata, India \\ kunalmahato34@gmail.com
}

\begin{abstract}
This paper is based on the concept of smart home automation. This paper aims to fulfil the needs of every person, especially the elderly or disabled ones, and it saves electricity at the same time. This system follows the home automation system concept and comes with a low cost and wireless network-based system. Features included in this system will change the standard of living at home.

This entire system will have remote access by a smartphone. At the same time, the system is made a safety control with a low voltage activation method. This system comes with a voice mode and realtime video streaming through an "Android application." It also helps the user to control the switches manually. This system is entirely user friendly, cost-efficient, and easy to install. The proposed system's prototype implementation is evaluated based on the criteria considered after the requirement analysis for an adequate home automation system.
\end{abstract}

Keywords: Arduino, IoT, Android, Wi-Fi, Home Automation

\section{INTRODUCTION}

The idea of "Home Automation" is not a new idea, rather it has existed from many years. The popular idioms like "Smart Home", "Intelligent Home" are introducing the concepts of networking appliances and devices in the house. A research area in engineering, and Computing opens by the designing of Home Automation Systems (HASs). HASs incorporate in different ways like, it is facilitating to lighting features, centralize control of appliances, security locks of gates and doors and lots of other additional systems, to provide advanced support to security systems and energy efficiency. HASs becoming well liked these days and join quickly in this emerging market. Moreover, end users, mainly the disabled and elderly attributable to their complexity and cost, don't always welcome these systems. For the improvement of wireless connectivity, there are a number of dissimilar of connections are launch such as GSM, WIFI, and
Bluetooth. Out of the four well liked wireless connections that provide implemented in HAS project, $\mathrm{Wi}-\mathrm{Fi}$ is being opt with its satisfactory capability. The capabilities of Wi-Fi are more than sufficient to be put into practice in the design.

Nowadays, most of the laptop/notebook or smartphone has built-in WIFI adapter. It will indirectly lower the cost of this system. In this paper authors have proposed the complete design of home automation and security system using Raspberry pi, a credit sized computer. Raspberry pi provides the same characteristics of a mini computer, and it's had the GPIO pins where other part and devices can be attaching. GPIO registers of Raspberry pi are used for the output grounds. Authors have designed a power strip that could be effortlessly connected to GPIO Pins of the Raspberry pi. The home appliances are connected to the input/output ports of Raspberry pi down with the power strip and their activity is observed to the raspberry pi. The android running Operating System in any phone connected to the network can enter the status of the home appliances via an application. It introduces the design and application of automation system that can monitor and command home appliances via android phone or tablet.

So, in this paper authors have tried to make the prototype of home automation system for controlling all the home appliances wirelessly with all the features including voice mode, video streaming and so on. An android device is made to to control the system with the help of an android device. The system can be used in wide range of areas.

\section{EXPERIMENTAL STEPS}

\section{A. Experimentation}

The first step for the development is the collection of necessary equipment and materials. Authors have first developed simple algorithms and flowcharts to complete the procedure.

\section{B. Design}

In this phase, authors have designed the layout of the application. The necessary features to be included. They 
were designing the power strip to connect the home appliances that can be controlled via GPIO pins.

\section{Developing and Testing}

In this phase, the development of application was performed. The bugs were identified and removed. We consulted many software experts for the evaluation of our application. Hardware design includes the design of power strip.

\section{Real World Testing}

Finally, our system was to be tested in the real electrical appliances.

\begin{tabular}{|c|c|c|c|c|c|c|}
\hline \\
\hline$g$ & & & & & & \\
\hline$\infty$ & & & & & & \\
\hline$\approx$ & & & & & & \\
\hline 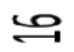 & & & & & & \\
\hline$\cong$ & & & & & & \\
\hline 戸 & & & & & & \\
\hline$\cong$ & & & & & & \\
\hline$\approx$ & & & & & & \\
\hline 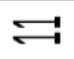 & & & & & & \\
\hline 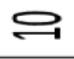 & & & & & & \\
\hline on & & & & & & \\
\hline$\infty$ & & & & & & \\
\hline r & & & & & & \\
\hline ט & & & & & & \\
\hline ת & & & & & & \\
\hline$\sigma$ & & & & & & \\
\hline$m$ & & & & & & \\
\hline$\sim$ & & & & & & \\
\hline$\longrightarrow$ & & & & & & \\
\hline 兽 & 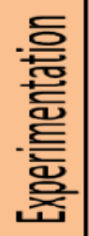 & 芸 & $\begin{array}{l}\text { 흘 } \\
\overline{\bar{y}} \\
\text { 흠 } \\
\text { 产 }\end{array}$ & 哭些 & 흥 & $\begin{array}{l}\text { 능 } \\
\text { 흘 } \\
\frac{\bar{w}}{\text { 흔 }}\end{array}$ \\
\hline
\end{tabular}

Fig. 1: Gantt Chart

\section{PROPOSED MODEL}

\section{A. Block Diagram}

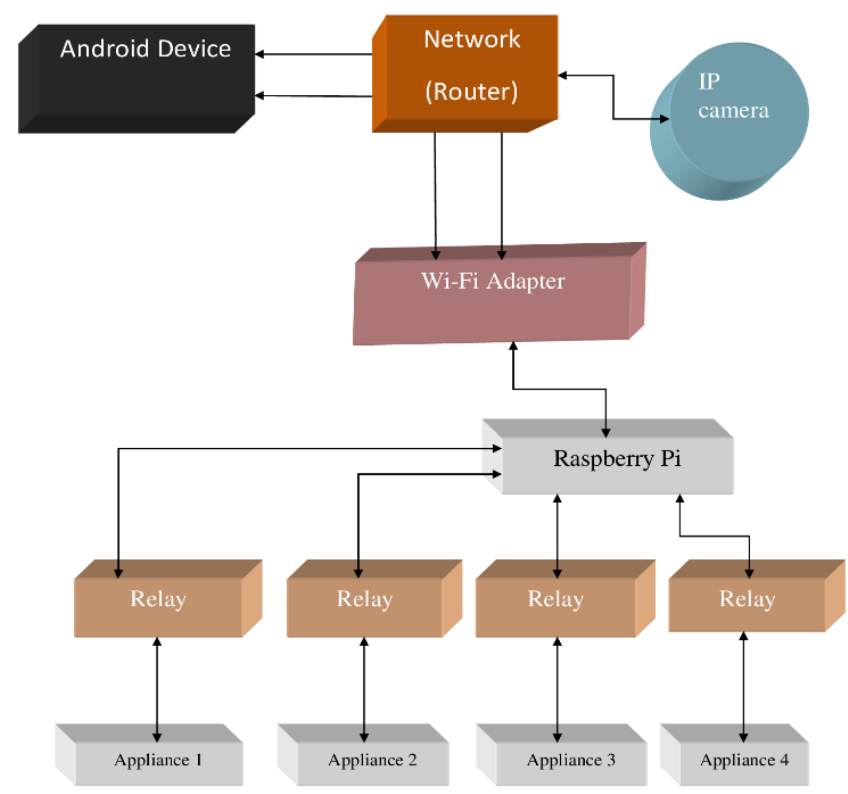

Fig. 2: Block diagram of Proposed system

\section{B. Proposed System}

The android operating system is an open source and provides a good platform to perform all the actions. The inbuilt sensors are easily accessible. The android device send all the data via programming and hence acts as a client.Our system is enriched with following features.

1) Switch Mode Activity.

2) Voice Mode Activity.

3) Video Mode Activity.

Switch mode will use the radio buttons that are used to control the home appliances wirelessly. The radio button also sends the status of the switch and we can switch the appliance on/off anytime. Moreover, manual control of all the switches will be present so as to make the entire process user friendly.

Voice Mode is used to control all the home appliances using voice command instructions. With the help of the inbuilt microphone of Smartphone or any other device, the application fetches the speech data to the Google server which responds with a string data. The string data are further analyzed and then processed accordingly.

Video Mode shows the live or recorded video stream of the room. The captured video will be streamed by the help of the android application. All the devices will be connected to a common network, through a common system. Smartphone, Raspberry pi and the IP camera are connected to the common network system. Router is used to create a common network and Wi-Fi Adapter is used to connect Raspberry pi to the network. Raspberry pi is used to maintain the server and the programming codes are uploaded to it in order to run the system. The Raspberry pi collects the data and further activates GPIO pins as necessary. The GPIO pins of raspberry pi are connected to the relays. Relay switches are used to 
connect the home appliances and it further takes actions according to the user's choice.

\section{IP Camera}

A security camera will give the freedom to have a home or business 24/7 surveillance via network anytime and anywhere. It also comes with alarm function, when somebody appears on the camera under alarm mode or alarm function, it will take a picture or activate the alarm and will give you an alert by emailing the pictures to you immediately.

\section{SOLUTION DETAILS}

\section{1) Hardware Environment}

\section{Control Electronics}

- Raspberry Pi as the controller for its processing power and large developer community.

- 4 Relays are connected to power strips.

- GPIO pins are connected to transistor. Transistors are used as switch.

2) Sofware Environment

\section{Android Developer Tools(ADT)}

-To build the android application to receive the live video feed from the camera and to send the signals to control the robot.

\section{RPI-GPIO library}

-GPIO interface library for the Raspberry Pi.

\section{APPLICATION DESCRIPTION}

The Android application consists of GUI which helps the user to control the appliances with an ease. The android application consists of various different activities as follows:
1) Start Mode
2) Option Mode
3) Voice Mode
4) Switch Mode
5) Video Mode

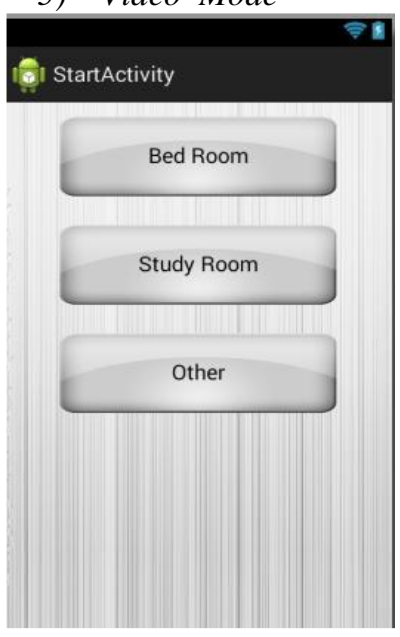

Fig. 3.1: Start Mode
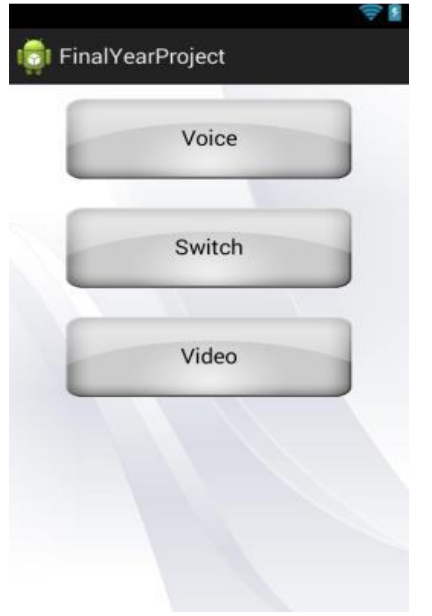

Fig. 3.2: Option Mode

\section{A. Start Mode :}

In this mode, all the rooms of the home will be displayed. The user can select the required room from the given options to control the appliances connected to specified room.

\section{B. Option Mode :}

The option mode provides the user with different types of usage options. The user can select either switch mode or voice mode to control all the appliances and can enjoy a real time surveillance through video mode.

\section{Voice Mode :}

The voice mode allows the user to give the speech instruction to the android application in order to control the appliance status. The speech data will be processed and required appliances are controlled accordingly.

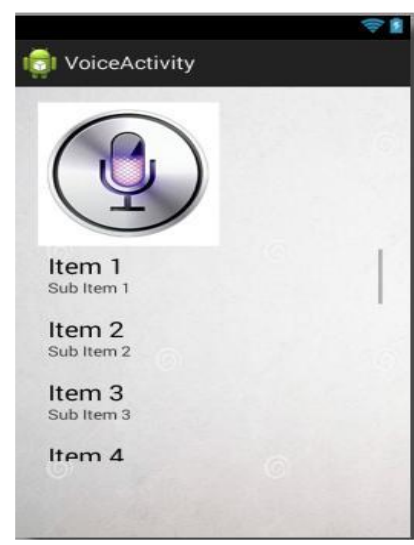

Fig. 3.3: Voice Mode

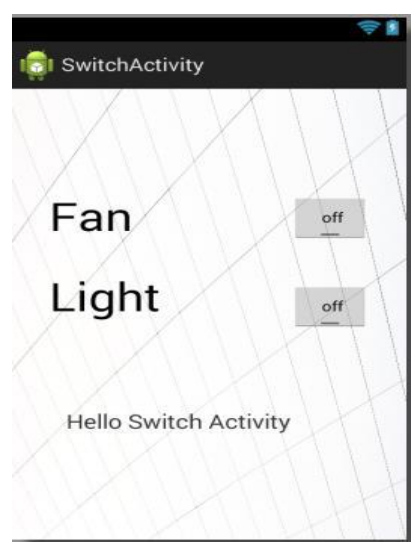

Fig. 3.4: Switch Mode

\section{Switch Mode :}

The switch mode helps the user to simply switch on/off the required home appliances with the help of buttons available in the android application.

\section{E. Video Mode :}

This mode displays the video of the IP cameras connected at the rooms of the home and user can enjoy a real time surveillance as well as the user can record video clips and can watch them later on.

\section{HARDWARE \& PROGRAMMING LANGUAGE}

\section{A. Hardware Details}

A power line will be designed and all relays will be connected to power line as well as all the home appliances will be connected to the power line. The Relays will be connected to the General Purpose input/output pins of the Raspberry pi. 


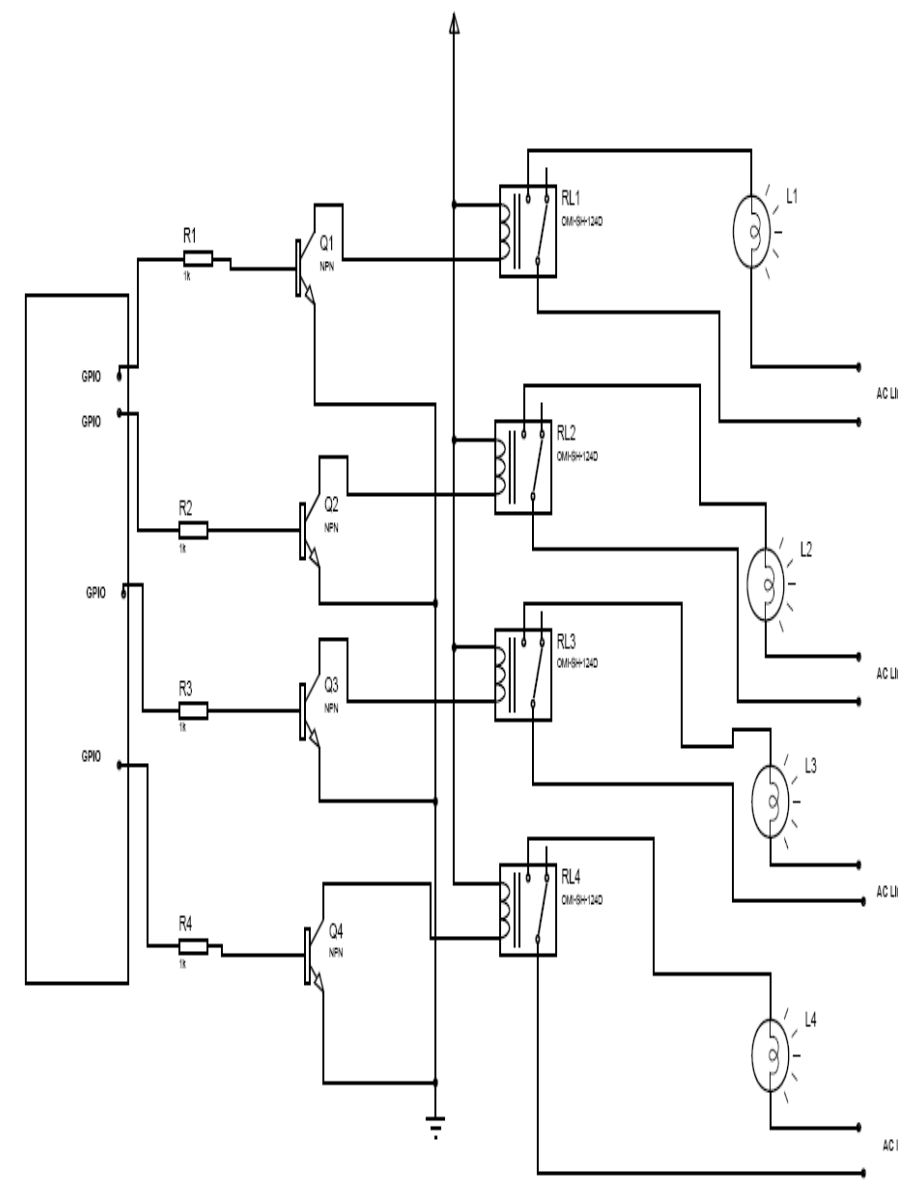

Fig. 4:Block Diagram of the Hardware Description

\section{B. Software Details}

Here we have used two different two different types programming languages for this project. For developing the app, we have taken taken the help of Android Studio. We have further used Java programming language for running the Android app. Eclipse software have been used to write codes under java environment. Raspbian operating system have been used for programming in Raspberry pi. The server had been setup at Raspberry pi. Python Langauage have been used to write the codes of the server and to take control of the Genereral purpose Input/Output pins of the operating system in Raspberry pi.

\section{PART LIST AND COST ESTIMATION}

TABLE 1. COST ESTIMATION

\begin{tabular}{|l|l|l|l|}
\hline Name & Number & Price & Availability \\
\hline $\begin{array}{l}\text { Raspberry Pi } \\
\text { 4 Model B } \\
\text { 2GB RAM }\end{array}$ & 1 & 3950 & Available \\
\hline $\begin{array}{l}\text { Wi-Fi } \\
\text { Adapter }\end{array}$ & 1 & 1500 & Available \\
\hline $\begin{array}{l}\text { SD card 32 } \\
\text { GB }\end{array}$ & 1 & 500 & Available \\
\hline Router & 1 & 2000 & Available \\
\hline $\begin{array}{l}\text { Relays(6V, } \\
\text { 3A, PCB } \\
\text { Mount) }\end{array}$ & 8 & 35 per piece & Available \\
\hline Jumper Wires & $1 \mathrm{pkt}$ & $\begin{array}{l}\text { Varies with } \\
\text { Quantity }\end{array}$ & Available \\
\hline Resistors & $1 \mathrm{pkt}$ & $\begin{array}{l}\text { Varies with } \\
\text { Quantity }\end{array}$ & Available \\
\hline Capacitors & $1 \mathrm{pkt}$ & $\begin{array}{l}\text { Varties with } \\
\text { quantity }\end{array}$ & Available \\
\hline
\end{tabular}

\section{CONCLUSION}

The main aim of this paper is to develop smartphone/tablet-based control system for all the home appliances effectively. The switch mode activity and voice mode activity are used to control the home appliances alternatively. The video feedback will be received in the android application which will stream the real time or recorded video of IP Camera.

The complete system is based on Raspberry pi, an Android platform supported by languages like Java and Python. The main advantage of this system is it is easy to develop and implementation cost of this system is also very low and can be easily configured according to user's choice.

Authors have developed a simple prototype and using this as a reference, the authors will further expand this work many other programs.

\section{ACKNOWLEDGMENT}

Authors wish to thank Prof. Sukalyan Goswami, HOD, Computer Science, UEM, Kolkata and all other departmental faculties for their ever-present assistant and encouragement. Last but not the least, we would like to extend our warm regards to all the group members and our families and peers who have kept supporting us and always had faith in our work. 


\section{REFERENCES}

[1] https://docs.python.org/

[2] http://developer.android.com/training/index.html

[3] https://docs.python.org/

[4] http://electronics.howstuffworks.com/

[5] http://elinux.org/RPi_Hub

[6] http://stackoverflow.com/

[7] http://www.raspberrypi.org/

[8] E. Yavuz, B. Hasan, I. Serkan and K. Duygu. "Safe and Secure PIC Based

[9] AmulJadhav, S. Anand, NileshDhangare, K.S. Wagh "Universal Mobile Application Development (UMAD) On Home Automation" MarathwadaMitraMandal's Institute of Technology, University of Pune, India Network and Complex Systems ISSN 2224-610X (Paper) ISSN 2225-0603 (Online)

[10] MohdHelmyAbdWahab, Muhammad IzharRamli, Nabihah, "TOWARDS SMART HOME: CONTROL ELECTRICAL DEVICES ONLINE",Nornabihah Ahmad International Conferenceon Science and Technology:Application in Industry and Education (2006).

[11] Remote Control Application for Intelligent Home”. International Journal of Computer Science and Network Security, Vol. 7, No. 5, May 2007.

[12] N. Sriskanthan and Tan Karand. "Bluetooth Based Home Automation System".

[13] Journal of Microprocessors and Microsystems, Vol. 26, pp.281289,2002 . 


\section{Indian Sign Language Classification (ISL) using Machine Learning}

\author{
Subhalaxmi Chakraborty \\ University of Engineering and \\ Management \\ Kolkata, India \\ subhalaxmi2008@gmail.com \\ Swatilekha Banerjee \\ University of Engineering and \\ Management, Kolkata \\ Kolkata, India \\ swatilekha123@hotmail.com
}

\author{
Nanak Bandyopadhyay \\ University of Engineering and \\ Management, Kolkata \\ Kolkata, India \\ nanakbandyopadhyay@hotmail.com \\ Zinnia Sarkar \\ University of Engineering and \\ Management, Kolkata \\ Kolkata, India \\ zinnia.rnj21@gmail.com
}

\author{
Piyal Chakraverty \\ University of Engineering and \\ Management, Kolkata \\ Kolkata, India
}

Sweta Ghosh

University of Engineering and

Management, Kolkata

Kolkata, India

ghosh.swetag@gmail.com

chakravertypiyal@gmail.com

\begin{abstract}
Communication is a crucial for humans, it is most vital. People with hearing or speaking disabilities need a way to communicate with other people of the society and vice versa. This paper presents a novel methodology in classifying the English Alphabets shown via various hand gestures in The Indian Sign Language (ISL) using Mediapipe Hands API, launched by Google. The objective of using this API is to detect 21 landmarks in each hand along with their $x, y$ and $z$ coordinates in $3 D$ space. Due to the scarcity of proper dataset available on the internet for ISL, at the very beginning, we have created a dataset having a size of 15000 , per English character, each consisting of the coordinates of 21 landmarks recognized by Mediapipe Hands API. From the literature, we found that prediction has been done for The American Sign Language and other foreign sign languages using Mediapipe API effectively. The novelty of our proposed work lies in using the same API for the Indian Sign Language. In this paper, we have discussed a comparative analysis of different classification algorithms like Support Vector Machine (SVM), Random Forest, Knearest neighbors (KNN), Decision Tree and other algorithms in terms of accuracy with the highest accuracy among all being $99 \%$. It is relevant to mention in this connection that the classification of the Indian Sign Language (ISL) using Mediapipe API is faster than the other conventional methods and outperforms in computational capability. This model can be used in web applications, mobile applications, desktop applications and in many more places.
\end{abstract}

Keywords - sign language, machine learning, computer vision, mediapipe, Indian sign language

\section{INTRODUCTION}

Communication is exceptionally pivotal to humans because it empowers us to express. The modes of communication include but are not limited to speech, body language, gestures, reading, writing etc. Unfortunately, there comes a communication gap for the speaking and hearing-impaired minority. Visual aids or an interpreter can be used in some cases to communicate with them [1]. Here the Sign Languages play an important role. These are virtual-gesture languages that are extensively used by deaf and hard-hearing people to communicate with others.

It has its own vocabulary that is distinct from that of spoken and written languages. Every word or alphabet is attributed to a certain gesture or body language (which entails movement of hands, limbs, or body to convey the speaker's thoughts) in sign language. [2]. Sign languages vary by area, such as American Sign Language (ASL), Indian Sign Language (ISL), and so on. This research focuses on Indian Sign Language. Indian Sign Language is mainly spoken in South Asian countries. ISL has a variety of qualities that set it apart from other sign languages. Number signs, family relationships, and the use of space are also essential elements of ISL. In addition, ISL has no temporal inflection [3]. The objective of the proposed work is to create a model that can correctly, easily, and consistently interpret alphabets in Indian Sign language based on their corresponding gestures in all lighting conditions. Recognizing sign language signs can be done in a variety of ways. Sanil et. Al used one of these methods: they trained a machine learning model to segment the skin portion of the image, extracted relevant features from the skin segmented images, used the extracted features as input into various supervised learning models for testing, and then used the trained models for classification. Sanil Jain and K.V.Sameer Raja used a number of approaches to train multiple machine learning models, with the maximum accuracy of 54.63 percent [4]. Rathna et. al used a different method of detecting hands to train the machine learning algorithm. They used the Microsoft XBOX360 Kinect Camera to obtain a dataset of depth-based segmented RGB images for classifying 36 distinct gestures. They used a Deep Convolutional Neural Network to identify the characters and got an accuracy of 89.30 percent [5]. This paper explores how one of Google's frameworks, Mediapipe Hands, can be used to detect hands with both speed and precision.

\section{BACKGROUND}

\section{A. Sign Language \& Gestures}

Sign language is a form of communicating for people who are deaf or hard of hearing. It's a series of hand signals, facial expressions, and body language that helps them to communicate with the rest of society. There isn't a single sign language. Rather, depending on the country, there are a number of sign languages around the world. Some countries, such as America, India, China, Mexico, Japan, Australia, and the United Kingdom, have their own sign language. Some gestures are performed with one hand, and others are performed with both hands [6]. According Hearing deficiency impacts 466 million people worldwide, with 34 million of them being teenagers, according to the World Health Organisation. WHO 
predicts that by 2050 , over 900 million people will have hearing damage that is incapacitating[7].

\section{B. Indian Sign Language}

India is a country rich in ethnic diversity as well as linguistic distinctions. Also for people with normal hearing and voice, communicating in a country like India is difficult. As a result, communication is much more complex for disabled persons. Also and, there are only a few schools that cater to them. Nonetheless, it falls short of meeting the needs of disabled people in this densely populated region. Furthermore, most rural areas have not yet been developed enough to provide opportunities for the deaf and dumb.

\section{Mediapipe Hands API}

Hands by MediaPipe (by Google) is a high-resolution hand and finger tracking solution. Machine learning (ML) is used to infer $213 \mathrm{D}$ landmarks of a hand from a single frame. MediaPipe's system achieves real-time efficiency on a mobile phone, and also scales to several hands, while existing state-of-the-art methods focus largely on strong desktop environments for inference.[8]

MediaPipe Hands makes use of a machine learning pipeline that consists of several models that work together:

A palm detection model that uses the entire image to generate an aligned hand bounding box. [8]

- A hand landmark model that returns high-fidelity 3D hand key points from the cropped image area identified by the palm detector.[8]

With proper inputs to the Mediapipe solutions API (discussed later), it provides the following output.

\section{- MULTI_HAND_LANDMARKS}

A series of detected/tracked hands, each of which is represented by a list of 21 hand landmarks, each of which is made up of the letters $\mathrm{x}, \mathrm{y}$, and $\mathrm{z}$. The picture width and height were used to normalise $\mathrm{x}$ and $\mathrm{y}$ to $[0.0,1.0]$. The landmark depth is represented by $\mathrm{z}$, with the origin being the depth at the thumb (landmark number 0 ), and the smaller the value, the closer the landmark is to the camera. The magnitude of $\mathrm{z}$ is measured on a scale that is similar to that of x. Fig 1 gives a visual representation of the hand landmarks.[8]

\section{MULTI_HANDEDNESS}

Collection of the detected/tracked hands' handedness (i.e., is it a left or right hand). Each hand is made up of two parts: a mark and a score. Label is a string with the value "Left" or "Right" in it. Score is the expected handedness's approximate likelihood, which is always greater than or equal to 0.5. (and the opposite handedness has an estimated probability of 1 - score). [8]
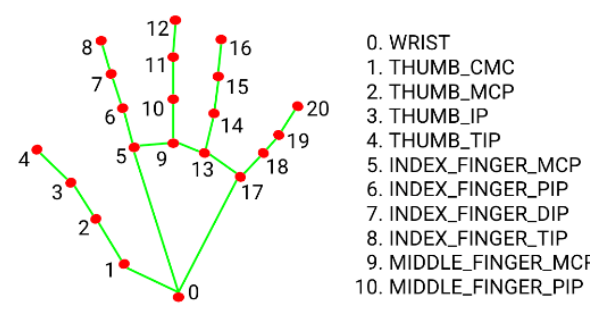

11. MIDDLE_FINGER_DIP 12. MIDDLE_FINGER_TIP 13. RING_FINGER_MCP 14. RING_FINGER_PIP 15. RING_FINGER_DIP 16. RING_FINGER_TIP 17. PINKY_MCP 18. PINKY_PIP 19. PINKY_DIP 20. PINKY_TIP

\section{Euclidian Distance and Slope of a line}

The length of a line segment between two points in Euclidean space is known as the Euclidean distance in mathematics.[9]

Let point $x$ and point $y$ have Cartesian coordinates $(x 1, x 2)$ and $(y 1, y 2)$ in the Euclidean plane respectively. The difference between $\mathrm{p}$ and $\mathrm{q}$ is then calculated as:

$$
d(x, y)=\sqrt{\left(y_{1}-x_{1}\right)^{2}+\left(y_{2}-x_{2}\right)^{2}}
$$

The slope of a line in the plane containing the $\mathrm{x}$ and $\mathrm{y}$ axes is defined as the change in the y coordinate divided by the corresponding change in the $\mathrm{x}$ coordinate between two distinct points on the line, and is commonly expressed by the letter $\mathrm{m}$ [10]. The following equation describes this:

$$
m=\frac{\Delta y}{\Delta x}=\frac{\text { vartical change }}{\text { horizonal chanos }}
$$

The difference in $x$ between two points $(x 1, y 1)$ and $\left(x_{2}, y_{2}\right)$ is $x_{2}-x_{1}$, while the change in $\mathrm{y}$ is $y_{2}-y_{1}$. The following equation is obtained by substituting all quantities into the above equation:

$$
m=\frac{y_{2}-y_{1}}{x_{m}-x_{1}}
$$

Euclidean Distance and the slope will be later used while creating the dataset.

\section{LITERATURE SURVEY}

There has been a lot of research into sign language recognition for American Sign Language (ASL), and there are many standard databases online. Indian Sign Language (ISL) research is still in its early stages. This may be attributed to ISL's low prevalence, rudimentary nature, and lack of recognition in society. Many variations remain between sign languages used in different areas due to a lack of standardization [10]. As a consequence, there is currently no standard dataset for ISL, so research is focused on existing datasets for other sign languages, or on generating or accumulating datasets from a variety of sources.

Hand gesture recognition can be performed using either vision-based or sensor-based methods [11].

- Vision Based: Vision-based techniques necessitate the use of a video camera to capture images or video of hand gestures. Three key stages comprise the highlevel pipeline for hand gesture recognition using a vision-based approach:

a) To distinguish the hand gesture from the image, this step includes clean-up and filter operations such as blurring, thresholding, image morphing, skin masking, and so on. This step is also know as the Image Preprocessing stage.

b) Feature extraction: This stage extracts features from the cleaned images. The image dataset is given a numerical representation. Since raw pixel data may be discordant and subject to noise, inversion, rotation, or illumination, feature extraction is necessary, making the learning process difficult. Feature extraction can be done

Fig. 1 Hand landmarks 
manually or automatically, using feature detection algorithms.

c) Classification: The image dataset's attributes serve as the learning model's training data. This knowledge can be used to train classification models such as SVM, Random Forest, K-nearest neighbours KNN, Decision Tree, and Neural Networks. The aplhabets can be predicted using the trained model.

- Sensor Based: This method necessitates the use of sensors and instruments to capture the hand's motion, direction, and velocity.

This method is brilliantly portrayed by $T$ Raghuveera, $\mathrm{R}$ Deepthi, $\mathrm{R}$ Mangalashri and $\mathrm{R}$ Akshaya [12]. They used Microsoft Kinect to collect images of the hand gestures. This gave them the advantage of being able to obtain depth information from the Kinect's infrared sensor in addition to RGB color information.

The use of sensors in Sign Language is very well explained by Edon Mustafa [14].

\section{PROPOSED WORK}

The aim of this research is to build a machine learning model that can rapidly and reliably identify Indian Sign Language movements. This machine learning model can then be used in a variety of ways to assist people with speech difficulties in communicating effectively. The objectives are described in more detail below.

- To develop or implement a system for recognising hand gestures that can be captured and used to train a machine learning algorithm.

- To make the dataset as wide and varied as practicable, since there is no readily accessible dataset for Indian Sign Language that can satisfy all of the criterias of the research.

- To prepare the dataset for the classification of different alphabets using various machine learning methods.

- To build a program that can detect hand movements and accurately identify the alphabet.

\section{WORKFLOW}

The diagram below illustrates the steps involved in

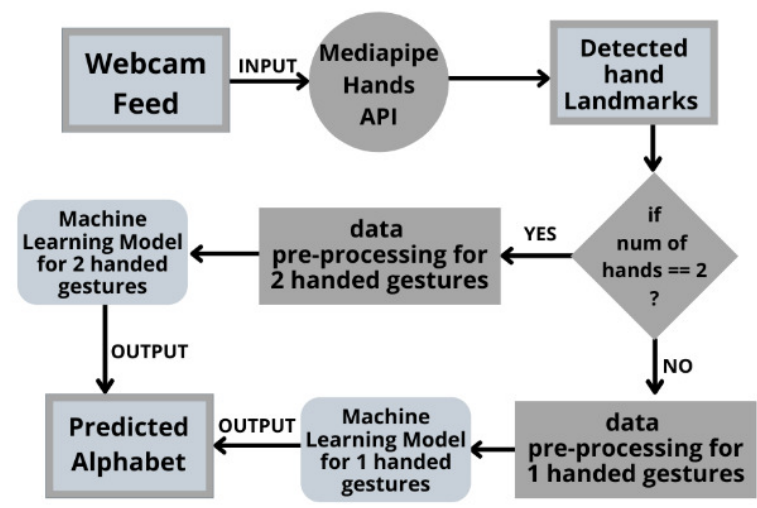

Fig. 2 Workflow diagram detecting the hands and developing a fully trained and working machine learning model for gesture detection at a high level.

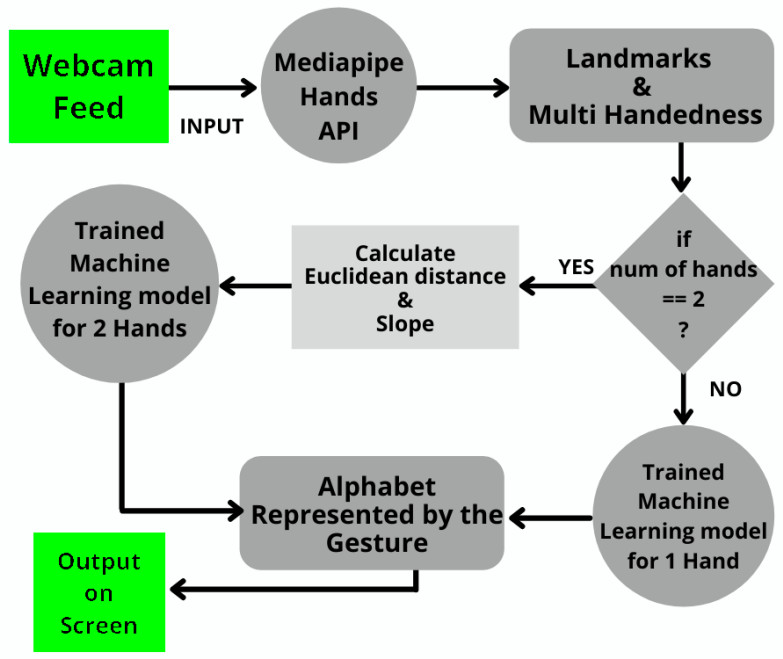

Fig. 3 Character prediction flowchart

\section{A. Hand Detection}

The palm detection model from Mediapipe handles the whole hand detection process. The webcam feed is fed into the

Mediapipe API, which returns a list of detected/tracked hands, each of which is defined by a list of 21 hand landmarks, each of which is made up of $\mathrm{x}, \mathrm{y}$, and $\mathrm{z}$. The landmark depth is represented by $\mathrm{z}$, with the origin being the depth at the thumb, and the smaller the value, the closer the landmark is to the camera. It also returns a list of the detected/tracked hands' handedness, as stated in section II (C).

\section{B. Dataset}

The dataset used for this work was made by the team themselves since there was no readily available dataset for ISL that fulfilled all the requirements of the work. As the Indian Sign Language consists of hand gestures that require the use of one as well as two hands, two separate datasets were made instead of one. One dataset contained all the data regarding the hand gestures that can be shown using only one hand and the other one had all the data

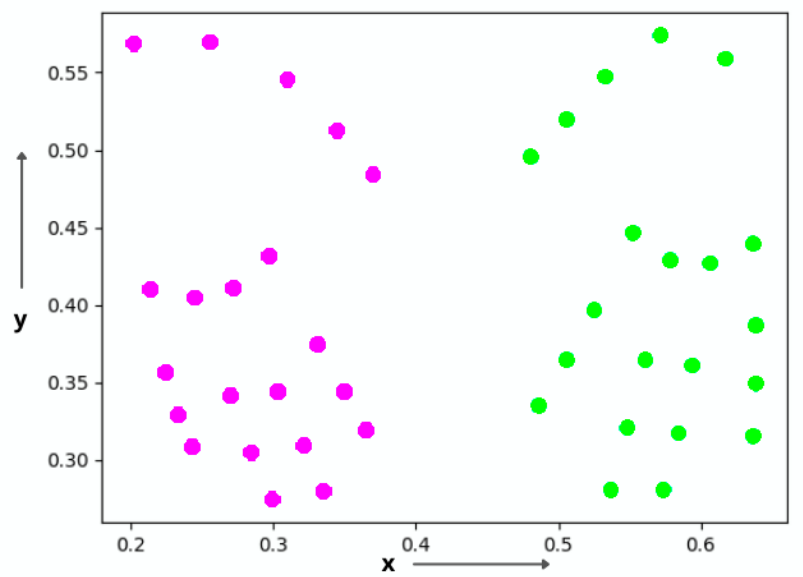

Fig. 4 Hand Landmark representation in 2D plane 
regarding the hand gestures requiring two hands. A python script was written for collecting the data from all the team members. The script was capable of detecting and extracting the landmarks of one or two hands using libraries like openCV and mediapipe. These landmarks consist of the $\mathrm{x}, \mathrm{y}$ and $\mathrm{z}$ coordinates as mentioned in section 3.1. The landmarks' coordinates were used accordingly for single-handed and double-handed gestures.

\section{1) Dataset for single-handed gestures}

Note - For this work, single-handed gestures are considered to be shown using only the right hand.

For each time a gesture was shown in the camera, the Mediapipe API returned the collection of handedness of the detected/tracked hands (i.e., is it a left or right hand) and collection of detected/tracked hands, where each hand is represented as a list of 21 hand landmarks and each landmark is composed of $\mathrm{x}, \mathrm{y}$ and $\mathrm{z}$. Using the count of detected hands, the python script written for data collection sends the data to the function that handles one handed gestures data pre-processing. Only the $\mathrm{x}$ and $\mathrm{y}$ coordinates are used from all the 21 landmarks detected by Mediapipe. These coordinates were inserted in a list in proper order preceded by the alphabet that is represented by the gesture. 15000 such records were dumped in the CSV file for each character.

Below is an example of how the single-handed gestures data looks.

\section{A list for one instance of the Alphabet $\mathrm{C}$}

$[\mathrm{C}, 0.94911146,0.62622416,0.85656536,0.6220784,0.770489$ $93,0.5701999,0.7059876,0.536494,0.63958037,0.5170507,0.825$ $8685,0.3889605,0.78857744,0.27900457,0.74550724,0.2527436$ $3,0.7029729,0.2544515,0.86787266,0.36955082,0.8263026,0.25$ $575498,0.776415,0.24780819,0.72802174,0.26539296,0.908185$ $66,0.37579635,0.8749907,0.2651433,0.8263938,0.24938047,0.7$ $778522,0.2553631,0.9495631,0.3972244,0.9301878,0.30835307$ $, 0.8907019,0.27846164,0.84412473,0.2635035]$

\section{2) Dataset for double-handed gestures}

Gestures that utilized two hands were in majority. Detecting these gestures and extracting the landmarks were done the same way as for the one-handed gestures. Only here the python script for data collection used the data in the function which handles pre-processing for two handed

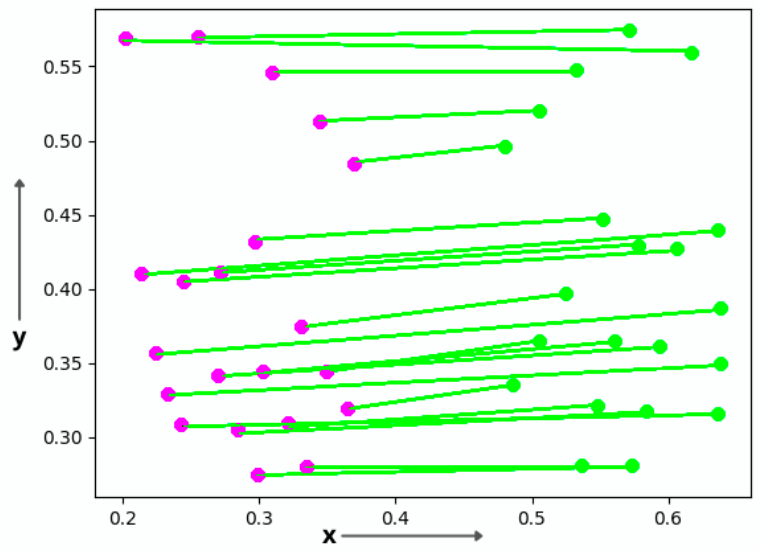

Fig. 5 Euclidean distance between similar landmarks gestures. The $\mathrm{z}$ coordinates were omitted here as well. The first approach for data collection was to create a list containing the coordinates of the landmarks for both the hands. Figure 3 represents the landmarks represented in 2D plane. This resulted in a huge dataset size. For each similar landmark of two separate hands, four values were inserted in the list. Each list for a single character had 85 elements in it including the character which the gesture represents. 15000 such lists had to be dumped in a CSV file which would result in a gigantic file size.

Better options were to be thought of. The figure below displays how Mediapipe detects the $\mathrm{x}$ and $\mathrm{y}$ coordinates and how they can be scattered in a 2D plane. The Euclidean distance between each similar landmarks of different hands were calculated as shown in figure 4.

The slope of those lines was calculated as well with respect to the $\mathrm{X}$-axis.

Thus now, for each similar landmark of two separate hands, only two values were calculated instead of four. This resulted for each list of a single gesture to consist of only 43 elements including the character that the gesture represents. Below is an example of two-handed gesture lists

\section{A list for one instance of the Alphabet A}

$[\mathrm{A}, 0.43240565,-0.118828712,0.28146366,-0.076334043$, $0.179769501,-0.024106368,0.11335197,-0.001396183$, $0.037270946,0.165703438,0.254766403,-0.011468012$, $0.217078681,-0.077803008,0.223184551,-0.018855066$, $0.239964854,0.001133083,0.337602944,-0.058893716$, $0.296307455,-0.057513888,0.30030761,-0.042237095$ $, 0.321719255,-0.015095411,0.420603901,-0.082614887$, $0.385677697,-0.094802589,0.377734235,-0.061607914$, $0.394410228,-0.030871268,0.508491004,-0.105871774$, $0.478781214,-0.121624107,0.449774288,-0.102564637$, $0.453709334,-0.072778826]$

\section{Training Machie Learning Models}

Both the datasets were trained separately using various machine learning models. The following description is given considering the dataset containing the all data regarding two handed gestures.

The dataset was divided into two parts, i.e. the training data set and the testing dataset in a ratio of a:b. Then the training data was used to train a Kernel SVM model, an SVM model, Random Forest classifier model, KNN classifier, Decision Tree, Naive Bayes and Logistic Regression. Exempt the Naive Bayes model and the Logistic Regression Model, all the other models worked exceedingly well.

\section{RESULTS AND DISCUSSION}

In this proposed work, using the mediapipe, prediction of the alphabets is done. Here, Fig. 6 displays a comparative analysis of the acquired results. Among Kernel SVM, SVM, Random Forest, KNN and Decision Tree, could have been used as all of these algorithms gave good results in their training phase. As the accuracy value is best in case Kernel SVM , this has been used. The webcam feed was given as input to the Mediapipe API and using it's returned value; it was decided whether the user was showing gestures using one hand or two hands. As mentioned earlier, most of the gestures required two hands and a few used only one hand. If the user was showing gestures using two hands, the Euclidean distance between 
the similar landmarks of different hands were found along with the slope that each of those lines made with the $\mathrm{x}$ axis. Then those distances and slopes were given to the trained Kernel SVM model and the predicted Alphabet was received as a returned value. On the other hand, if the user displayed a hand gesture using only one hand, the landmarks of that hand gesture would be sent to the trained Kernel SVM model for

\section{Accuracy Comparison}

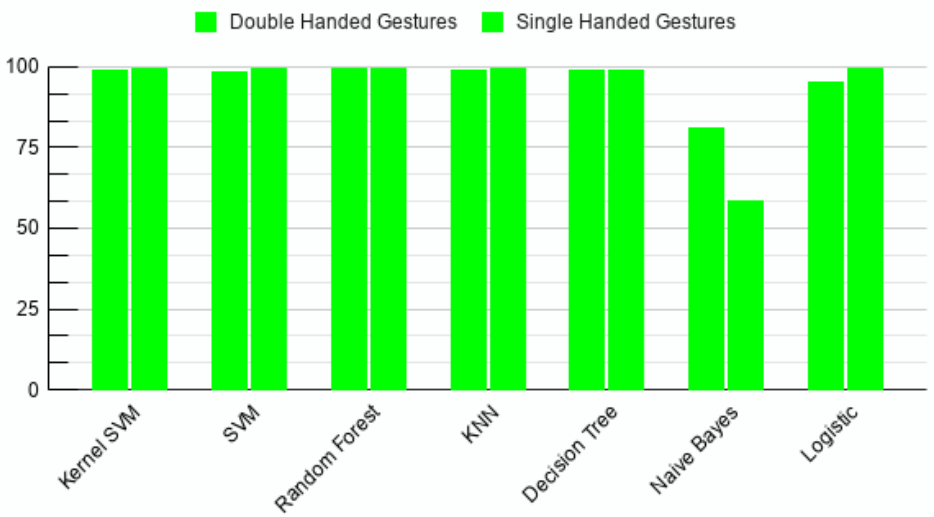

Fig. 6 Accuracy Comparison between Single and Double handed Signature

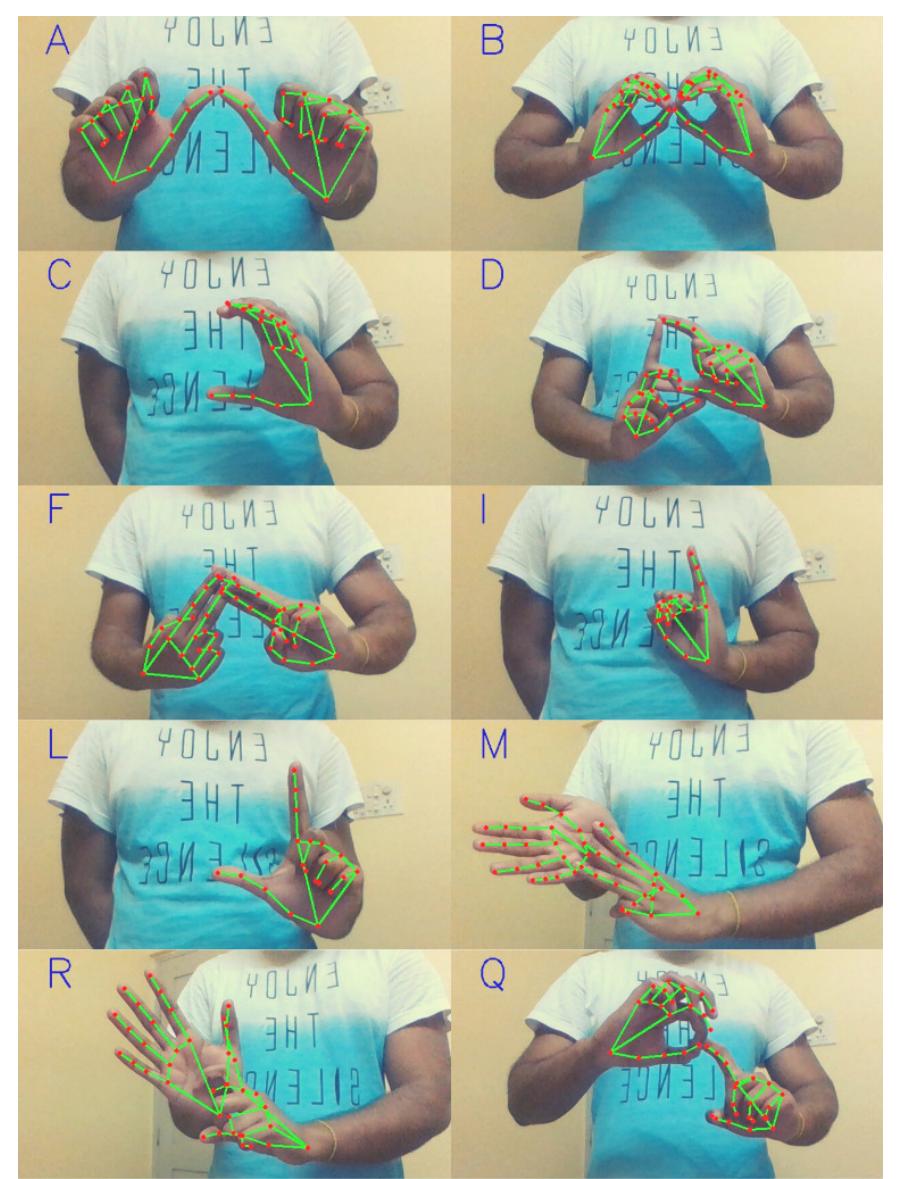

Fig. 7 Predicted alphabets one hand gestures. The returned values were the predicted alphabets which were displayed on the screen. Fig. 7 shows the predicted output of hand gestures.

\section{FUTURE SCOPE}

This initiative seeks to make connectivity simple for all and will benefit from the following:

- The trained machine learning model can be integrated in a web or smartphone framework to provide an end-to-end solution that is simple to use for all.

- Other sign languages, in addition to Indian Sign Language, may be added. A functionality that encourages the user to incorporate sign languages on their own will greatly improve the accessibility and user experience.

\section{REFERENCES}

[1] Muskan Dhiman and Dr G.N. Rathna on SIGN LANGUAGE RECOGNITION, Department of Electrical Engineering, DSP Lab, Indian Institute of Science, Bangalore

[2] Ashok Kumar Sahoo on Indian sign language recognition using neural networks and kNN classifiers, August 2014, Journal of Engineering and Applied Sciences 9(8):1255-1259

[3] Dewang Sultania, "Indian Sign Language Recognition", unpiblished.

[4] Sanil Jain and K.V.Sameer Raja, "Indian Sign Language Character Recognition", Indian Institute of Technology, Kanpur.

[5] Paranjoy Paul and Dr. G N Rathna, "Real time Indian Sign language recognition", in Summer Research Fellowship Programme of India's Science Academies.

[6] Sign Language Alphabets From Around The World, www.aimedia.tv

[7] Deafness and hearing loss, www.who.int

[8] Mediapipe Hands, google.github.io/mediapipe/solutions

[9] Tripathi, K., \& Nandi, N. B. G. C. Continuous Indian Sign Language Gesture Recognition and Sentence Formation. Procedia $\begin{array}{lll}\text { Computer } & \text { Science, } & 54,\end{array}$ 531. doi:10.1016/j.procs.2015.06.060,2015

[10] R. Johnson and J. Johnson, "Distinction between West Bengal Sign Language and Indian Sign Language Based on Statistical Assessment”, Sign Language Studies, vol. 16, no. 4, pp. 473-499, 2016. Available: https://www.jstor.org/stable/26191231.

[11] M. Cheok, Z. Omar and M. Jaward, "A review of hand gesture and sign language recognition techniques", International Journal of Machine Learning and Cybernetics, vol. 10, no. 1, pp. 131-153, 2017. Available: $10.1007 / \mathrm{s} 13042-017-0705-5$

[12] Raghuveera, T., Deepthi, R., Mangalashri, R. et al. "A depth-based Indian Sign Language recognition using Microsoft Kinect". Sādhanā 45, 34 (2020). https://doi.org/10.1007/s12046-019-1250-6

[13] R. Johnson and J. Johnson, "Distinction between West Bengal Sign Language and Indian Sign Language Based on Statistical Assessment", Sign Language Studies, vol. 16, no. 4, pp. 473-499, 2016. Available: https://www.jstor.org/stable/26191231.

[14] Mustafa, Edon \& Dimopoulos, Konstantinos. (2014). "Sign Language Recognition using Kinect". Avaiable: https://www.researchgate.net/publication/266144236_Sign_Langua ge_Recognition_using_Kinect 


\title{
Fake Note Detection using Machine Learning Techniques
}

\author{
Subhalaxmi Chakraborty, Soumyadip Banerjee, Biman Kumar Singha, SayaniGhati \\ Department of Computer Science and Engineering, UEM Kolkata
}

\begin{abstract}
In recent scenario detection of fakenote has become a genuine problem in the area of the financial sector as per the of various countries. In this paper, we have proposed a machine learning model that is capable of eradicating the fake note problem. In this paper, we have used a dataset of fake note images having a size of 1500 . Hence exhaustive experiments have been conducted using various machine learning algorithms for proper authentication of the banknote. Here we considered K-Nearest Neighbour, Naive Bayes and random forest classifier technique yielding various result in terms of accuracy, precision and recall and f-score. It is observed that the $\mathrm{K}$ - nearest neighbour technique shows better performance compared to the other applied algorithm having an accuracy of $99 \%$. Moreover, it is observed that it gives a result on determining whether a note is fake or real by output 0 when the note is fake and it gives output 1 when the note is real. Hence $\mathrm{K}$ nearest neighbour gives there result more accurately than other classifiers. The rules are given by machine learning classifier techniques also tested and found that they are accurate enough to be used for prediction and compare their performance to see which classifier performs best on determining the fakenote and showing their performance by bar-graphrepresentation.
\end{abstract}

Keywords - KNN , Naive Bayes ,Random Forests, Accuracy, Precision, Fake note

\section{Introduction}

In recent days machine learning techniques are tremendously used in fake note detection. Kim et al. applied both ensemble method boosting for improving the performance of traditional neural network in bankruptcy prediction and concluded that ensemble boosted neural networks performance is better than traditional neural networks [1]. The experimental method has set up using a method that Hold on method. The method is one in which the dataset is separated into subsets (70:30ratio) called the training set and testing set respectively. The training set is used to train the classifier while testing set is used to estimate the error of trained classifier [2]. A naive Bayes classifier is used when features are independent to each other in each class. It is based on estimating $\mathrm{P}(\mathrm{X} \mid \mathrm{Y})$, probability of $\mathrm{X}$ given that $\mathrm{Y}$ is occurred. It classifies the process into training and prediction step [3]. Sun et al. applied support vector machine for financial distress prediction. SVM is a supervised machine learning technique that uses classification algorithm for two group classification problems. SVM helps to finding out more accurate result towards class prediction[4]. Danenas et al. applied linear SVM for credit risk evaluation to show that it performs better than logistic regression in terms of accuracy [5]. Boyacioglu et al. applied ML, $\mathrm{K}$ means cluster, learning vector quantization etc. Those techniques helps the model far better for banknote detection purpose [6]. Preserve genuinity of printed banknotes is one of the critical thing. It has a major role in financial activities of a country. Aoba et al. used three layered perception and euro banknote recognition with various $\mathrm{RBF}$ Ker tools [7]. Zhou et al. has done the performance evaluation of corporate fake note prediction by imbalanced dataset by applying sampling method. Sampling method gives more accuracy to find out the prediction about fake note [8]. Yu et al. applied Leave-One-Out incremental extreme machine learning for bankruptcy prediction and has given specific financial indicator [9]. Guangli et al. hasapplied decision tree and logistic regression to performs better. Decision tree helps to find out the prediction of fake note more accurately and gives desired output. Logistic Regression also performs well that estimates the probability of class ownership [10]. The dataset used for carrying out the experiments is taken from UCI machine learning dataset that gives huge number of entries of dataset for bankruptcy prediction with total five attributes [11]. Zhang et al. applied 
logistic regression and artificial neural network for bankruptcy prediction and it shows ANN performs better than LR and Logistic Regression is not that much accurate as like as Artificial Neural Network [12].Mayadevi A. Gaikwad et al. has done automatic fake currency detection technique using image processing. They have used coins, banknote and electronic data as currency and also the idea of image segmentation and characteristic extraction has been applied for achieving better performance[13].Renuka Nagpureet al. has applied image processing and java-based application that helps to recognize a bank note based on its denomination on an application window. They have also worked for various image recognition method that efficiently helps in currency recognition and fake note detection[14]. M.Deborahet al. has applied some image enhancement techniques that contains image segmentation, cropping, smoothing, contrast stretching, de-blurring and adjusting for finding fake currency. They have also used image acquisition, edge detection and Peak Signal to Noise Ratio (PSNR)technique to identify fraudulent currency [15]. Faiz $M$. Hasanuzzaman et al. has done banknote recognition based on robust and effective component. They have used a camera-based computer vision technology to automatically recognize banknotes for assisting visually impaired people. SURF technique is applied there to repeatability, distinctiveness and robustness of local image feature extraction in banknote recognition [16]. Mohammad $\mathrm{H}$ Alshayeji et. al. has done counterfeit currency detection using bit- plane slicing technique. This technique consists of decomposing original images of 256 grey levels into their equivalent 8 binary images. They have used MATLAB function for edge detection, image acquisition etc. [17].Komal Vora et. al. has done currency recognition system based on frequency domain feature extraction method and implementation of OCR. An optimal and efficient implementation of two-dimensional discrete wavelet transform to develop a currency recognition system and non-textural features are used for checking authenticity. The concept of histogram equalization is also used for better result [18].Ankush Singh et al. has done fake currency detection using image processing and cloud storage and taken images of currency for detection. They have applied a proposed solution in form of an mobile app coupled with cloud storage. Image processing algorithms are applied to extract the features such as security threat. SVM or Support Machine Vector algorithm is used for better result [19]. Devid Kumar et al. has applied computer vision technology and feature extraction in fake currency detection. They mainly have used ORB (Oriented Fast and Rotated BRIEF) and Brute-Force matcher approach to extract the feature of paper currency. Some sort of image processing techniques and feature extraction algorithms are applied to detect fake currency [20]. M. Laavanya et al. has applied deep learning and image processing technique on real time fake currency detection to avoid less efficiency and time consuming. They have used the concept of deep convolutional neural network and feature extraction. A transfer learning using Alex net that is popular in deep learning is adopted for detecting fake currency [21]. Navya Krishna et al. has applied convolutional neural network for designing Automatic Fake Currency Recognition system(AFCRS) that performs better than previously used image processing techniques. They have trained an artificial neural network and make a neural network to predict a class in that an image belongs to. VGGNet in $\mathrm{CNN}$ is chosen for the model to perform better [22]. Dr. P. Mangayarkarasi et al. applied image acquisition, feature extraction and comparison method for recognizing fake Indian currency note. They have produced the result in the form of text and voice by using image segmentation and edge detection technique. Brute Force Classification algorithm is used to calculate hamming distance using the descriptor that returns the point with minimum hamming distance applied on the notes [23]. Snehlataet al. used Principle Component Analysis (PCA) technique and image pre-processing for fake currency detection. PCA is used to detect the feature of currency through modelling. PCA is a method of identification of data patterns in that data are expressed in order to highlight 
similarities and difference. MATLAB is used for numerical computation and image processing[24].P. Gayathri et al. used texture classification and some image processing methods and convolutional neural network for fake Indian currency detection. They have applied the concept of ANN and CNN to make result and performance more accurate and efficient. The usage of image re-scaling and image shearing makes it more efficient and helps to gaining desired result [25]. The goal is to detect a note is fake or not. This thing is occurred in banking purpose and other economical aspects. In this Paper we have to take three machine learning classifiers to train a dataset and then we test a note by this classifier to check it is fake or not. The entire dataset has been divided into 70:30 ratio means $70 \%$ data is used for training purpose and remaining 30\% data is used for testing purpose. The same thing is happening with those three classifiers and finally we observe which classifier predicts at its best by measuring classifier performance. We comment on best classifier and represent the bar- graph according to classifiers performance. So we used some packages like Sklearn, matplotlib and functions like Gaussian NB, RFC, KNN etc. in python programming language. Here data pre- processing is used before applying classification techniques on dataset that cleans out the entire data from missing values and null values. For preprocessing some python module has been used and some particular mean strategy is applied for pre-processing that replace all missing or null values with the value of mean of that particular column of the dataset.

\section{Description of Dataset}

The dataset used for fake note detection is taken from UCI dataset. This dataset has total 1372 instances. The dataset has 5 attributes. Among five first four attributes are used as input data and the last column that is used for target purpose means the output column. Those first four columns are Variance, Skewness, Kurtosis, Entropy. The last column "Class" describes the value ' 0 ' and ' 1 ' where ' 0 ' describes the note is real against the input data and if it is ' 1 ' that describes the note is fake.
Variance: - It is a measure of 'spread' of a distribution about its average value.

Skewness: -Skewness tells about the direction of variation of the lack of symmetry.

Kurtosis: - Kurtosis is a parameter that describes the peakof distribution.

Entropy: -Image entropy is the amount of information which must be coded for bya compression algorithm.

Class: - Class contains two values that is 0 and 1 where 0 represents real banknote and 1 represent fake note.

Excluding the 'class' remaining four attributes represents the input of a note that is given to check it is fake or real and the target column represents the output.

\section{Method of problemsolving}

This experiment is performed using a method in machine learning called classification. During classification three different types of algorithm is used to find the output. The total dataset that is used for the measurement of fake note detection is divided into two subsets in 70:30 ratio. First subset is training set and the other one is testing set. Training set is used to train the dataset to gain experience and the testing set is used to check the performance and estimate the error rate. Before applying some classification algorithm some data preprocessing is taken into consideration.

\section{i) Datapre-processing}

Some packages are used to pre-process the dataset before applying some classification technique. 'simpleimputer' package is used for pre-processing. It is used to replace the missing or null values with the average of the values present in that column. It is used to avoid errors duringmeasurement.

ii) Performing classificationtechniques

Three classification techniques are used like Random forest, KNN and naive Bayes classifier. For each classifier dataset is divided into two subsets and then applying different techniques. Then testing the note against some input data to measure the performance of classifier and find the outcomes. After that some metric values are find out.

iii) Performancemeasure 
To measure the performance of every classifier some metric values are taken into consideration like accuracy, precision, recall and F1- score. Accuracy $=(\mathrm{TP}+\mathrm{TN}) /(\mathrm{TP}+\mathrm{TN}+\mathrm{FP}+\mathrm{FN})$ Accuracy is defined as the percentage of correct predictions for the test data. It is calculated by dividing the number of correct predictions by the number of totalpredictions.

Precision $=\mathrm{TP} /(\mathrm{TP}+\mathrm{FP})$

Precision is the ratio of true positives to the total of the true positives and false positives.

Recall $=\mathrm{TP} /(\mathrm{TP}+\mathrm{FN})$

Recall quantifies the number of positive class predictions made out of all positive examples in the dataset.

F1-score $=2 *(($ Precision $*$ Recall $) /($ Precision + Recall))

F1-score is the harmonic mean of precision and recall and gives a better measure of the incorrectly classified cases than the accuracy metric.

$\mathrm{TP}=$ True Positive $\mathrm{TN}=$ True Negative FP $=$ False Positive FN = False Negative

\section{iv) PerformanceRepresentation}

The all metrics from each classifier are taken and represented in bar graph which clearly determines the performance of every classification technique.

\section{Machine Learning Classifiers Techniques}

There are three different classifier techniques are used as mentioned below:

\section{1) Random Forest Classifiertechnique}

Random forest is a supervised learning algorithm which is used for both classification as well as regression. But however, it is mainly used for classification problems. Like a forest have many trees, a random forest algorithm creates decision trees on data samples and then gets the prediction from each of them and finally selects the best solution by means of voting. It is an ensemble method which is better than a single decision tree because it reduces the overfitting by averaging the result. The greater number of trees in the forest leads to higher accuracy and prevents the problem of overfitting. It takes less training time as compared to otheralgorithms. For classification purpose, random forest algorithm required Gini index formula that is used to decide how nodes on a decision tree branch.

$$
\text { Gini }=1-\sum_{i=1}^{c}\left(p_{\mathrm{i}}\right)^{2}
$$

This formula uses the class and probability to determine the Gini of each branch on a node, determining which of the branches is more likely to occur. Here, pi represents the relative frequency of the class that is observing in the dataset and c represents the number of classes. Entropy is also used to determine how nodes branch in a decision tree.

Entropy $=\sum_{i=1}^{c}-p_{\mathrm{i}} * \log _{2}\left(\mathrm{p}_{\mathrm{i}}\right) \ldots$. (ii)

\section{2) Naive Bayes Classifiertechnique}

Naive Bayes classifiers are a collection of classification algorithms based on Bayes' Theorem. It is not a single algorithm but a family of algorithms where all of them share a common principle, means every pair of features being classified is independent of each other. A Naive Bayes classifier is a probabilistic machine learning model that's used for classification task. The crux of the classifier is based on the Bayes theorem. It is mainly used in text classification that includes a high- dimensional training dataset. Naïve Bayes Classifier is one of the simple and most effective Classification algorithms which helps in building the fast machine learning models that can make quick predictions. It is a probabilistic classifier, which means it predicts on the basis of the probability of an object. The formula of Baye's theorem is given by :

$\mathrm{P}(\mathrm{A} \mid \mathrm{B})=\frac{P(B \mid A) P(A)}{P(B)}$

$\mathbf{P}(\mathbf{A} \mid \mathrm{B})$ is Posterior probability: Probability of hypothesis $\mathrm{A}$ on the observed event $\mathrm{B}$.

$\mathbf{P}(\mathbf{B} \mid \mathbf{A})$ is Likelihood probability: Probability of the evidence given that the probability of a hypothesis is true.

$\mathbf{P}(\mathrm{A})$ is Prior Probability: Probability of hypothesis before observing theevidence.

$\mathbf{P}(\mathbf{B})$ is Marginal Probability: Probability of Evidence.

The formula of Baye's theorem in following way: 
$\mathrm{P}(\mathrm{y} \mid \mathrm{X})=\frac{P(X \mid y) P(y)}{P(X)} \ldots(\mathrm{iv})$

Here, $\mathrm{y}$ is class variable and $\mathrm{X}$ is a dependent feature vector where: $X=(x 1, x 2, x 3 \ldots \ldots .$. , $\mathrm{xn})$

$\mathrm{P}\left(\mathrm{y} \mid \mathrm{x}_{1}, . ., \mathrm{x}_{\mathrm{n}}\right)=$ $\frac{P(x 1 \mid y) P(x 2 \mid y) \ldots \ldots P(x n \mid y) P(y)}{P(x 1) P(x 2) \ldots . . P(x n)} \ldots \ldots \ldots \ldots$.

That can be expressed as:

$\mathrm{P}\left(\mathrm{y} \mid \mathrm{x}_{1}, \ldots . ., \mathrm{xn}\right)=$

$\frac{P(y) \prod_{i=1}^{n} P(x i \mid y)}{P(x 1) P(x 2) \ldots . P(x n)}$

\section{3) KNN classifiertechnique}

KNearestNeighbour is one of the simplest Machine Learning algorithms based on Supervised Learning technique. KNN algorithm assumes the similarity between the new case/data and available cases and put the new case into the category that is most similar to the available categories. KNN algorithm stores all the available data and classifies a new data point based on the similarity. This means when new data appears then it can be easily classified into a well suite category by using KNN algorithm.It belongs to the supervised learning domain and finds intense application in pattern recognition, data mining and intrusion detection. For performing KNN algorithm Euclidean distance is taken to find out distance between testing data point to each and every training points.

Distance

$\mathrm{D}=\sqrt{\sum_{i=1}^{k}(x i-y i)^{2}}$.

Here, $\mathrm{k}$ is the distance between $\mathrm{k}$ data points.

\section{4) Data Pre-processing}

Data pre-processing is a process of preparing the raw data and making it suitable for a machine learning model. It is the first and important step while creating a machine learning model. A realworld data generally contains noises, missing values, and maybe in an unusable format which cannot be directly used for machine learning models. Data pre-processing is required tasks for cleaning the data and making it suitable for a machine learning model which also increases the accuracy and efficiency of a machine learning model. At first the dataset is taken at the format of .csv file. That dataset may contain some missing values and noises which may affect the overall calculationsandotheraspects. Somepackageisusedfordata pre-processing like "simple imputer". Pre-procesing refers to the transformations applied to our data before feeding it to the algorithm.

In other words, whenever the data is gathered from different sources it is collected in raw format which is not feasible for the analysis.For achieving better results from the applied modelin Machine Learning Papers the format of the data has to be in a proper manner. Some specified Machine Learning model needs information in a specified format, for example, Random Forest algorithm does not support null values, therefore to execute random forest algorithm null valueshave

tobemanagedfromtheoriginalrawdataset.Aotheras pectisthat data setshould be formatted in such a way that more than one Machine Learning and Deep Learning algorithms are executed in one data set, and best out of them is chosen. Mean strategy is taken to fill out those missing values to make the dataset without null values that prevents various kinds of data hazards

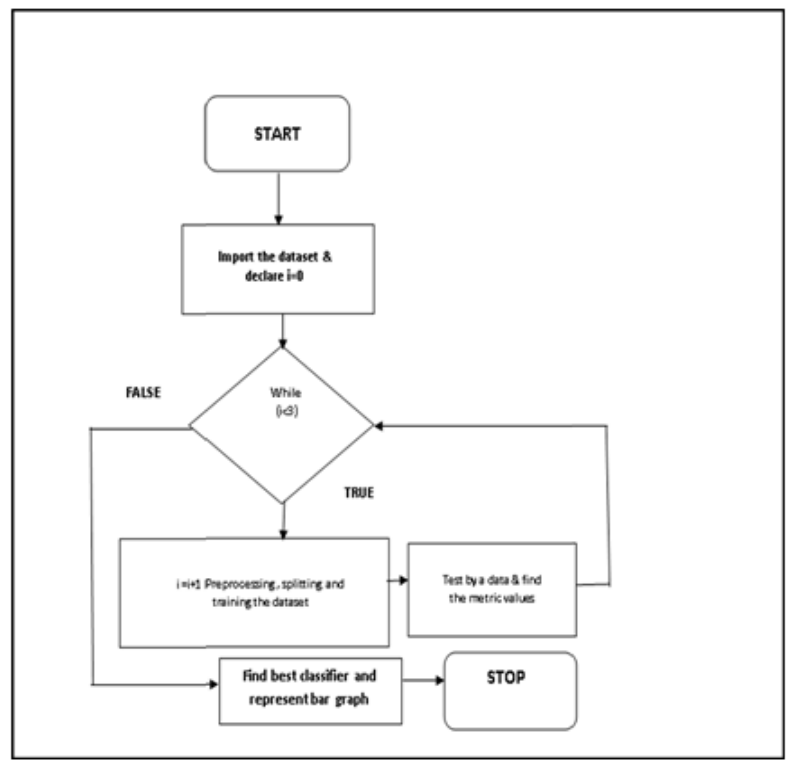

and data duplicity.

Fig. 1. Flow Chart for Fake note Detection 


\section{algorithm}

\section{Result andDiscussion}

After performing three types of classification algorithm on the dataset the performance of those classifiers are different from each other. The result of three classifiers is shown below. From the below mentioned table, it is seen that KNN classifier gives highest accuracy $99.76 \%$ that is higher than other two classification technique. The Naïve Bayes Classifier have the ccuracy of $85.92 \%$ and Random Forest classifier has $99.27 \%$ accuracy that is nearest to the KNN classifier.F1-score value is found out from accuracy, precision and recall that determines which classifier performs best. From the result of those different classifier techniques a bar graph is obtained that tells which classifier performs best.

Table: I: Comparative analysis of various classification techniques

\begin{tabular}{|l|c|c|c|}
\hline $\begin{array}{c}\text { Technique } \\
\text { name }\end{array}$ & Accuracy & Precision & Recall \\
\hline $\begin{array}{l}\text { RandomForest } \\
\text { classifier }\end{array}$ & $99.27 \%$ & $98.85 \%$ & $99.42 \%$ \\
\hline $\begin{array}{l}\text { Naïve } \\
\text { Bayesclassifier }\end{array}$ & $85.92 \%$ & $84.22 \%$ & $82.29 \%$ \\
\hline $\begin{array}{l}\text { KNN } \\
\text { classifier }\end{array}$ & $99.76 \%$ & $99.50 \%$ & $99.8 \%$ \\
\hline
\end{tabular}

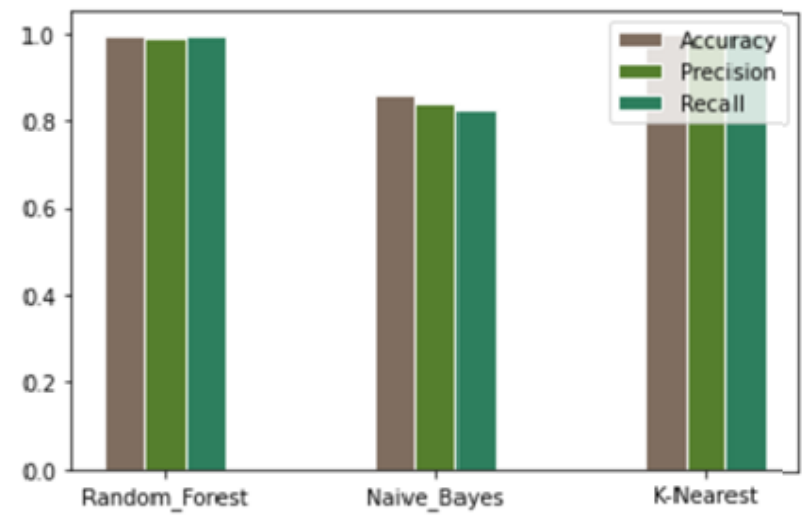

Fig . 2. Performance Measurement of various classification techniques in graphical representation

The bar graph represents the performance percentage ofRandom Forest, Naïve Bayes and KNN classifier in terms of accuracy, precision and recall. It has a scale between 0 to 1 with having an interval of 0.2 that helps to measure the performance metrics of each classifier. Three different colours are associated for accuracy, precision and recall. This graph helps to visualize the performance of each classifier and also helps to determine the classifier that performs best. Those three classifiers give the same output against particular input data of a given note and according to accuracy and other metrics they perform differently. The four input values thoseare separated by comma represents the values of four attributes of a note. Those are Variance, Skewness, Kurtosis and Entropy respectively. Output represents the prediction of the class ofnotedepending on four input values. The class is 1 that determines the note isfake. According to the bar-graph, the performance of $\mathrm{KNN}$ classifier is best than other two.

\section{Conclusion and Future Scope}

In this proposed work of fake note detection using various machine learning classification technique we analysed on the basis of Random Forest, Naïve Bayes, KNN. So, it works fine against all input of banknote and gives the expected result that whether the note is fake or real. Moreover, we determine which classifier performs better by finding metric values like accuracy, precision and recall represent the bargraph by those values with respect to the performance of classifiers. In this regard, we obtained that KNN classification shows better result terms of accuracy compared to other models used, based on same fake note dataset. This work can be extended in the field of latest technologies like deep neural network, texture classification, convolutional neural network, information retrieval etc.

\section{References}


[1] Myoung-Jong-Kim, Dae-Ki Kang, Ensemble with neural networks for bankruptcy prediction, Expert Systems with Applications 37 (2010) 3373-3379.

[2] https://raw.githubusercontent.com/lucko515/ classification-

python/master/Banknotes\%20Authentication\%2 0Classification/data

anknote_authentication.csvaccessed

on $(5 / 5 / 2020)$.

[3] Anamika Yadav, Aleena Swetapadma, Combined DWT and Naive Bayes Based Fault Classifier for protection of Double Circuit Transmission Line, IEEE International Conference on Recent Advances and Innovations in Engineering(ICRAIE-2014), May 09- 11, 2014, Jaipur India.

[4] Jie Sun, Hui Li, Financial distress prediction using support vector machines: Ensemble vs. Individual, Applied Soft Computing 12(2012) 2254-2265.

[5]PauliusDanenas, Gintautas Garsva, Selection of Support Vector Machines based classifiers for credit risk domain, Expert Systems with Applications 42(2015) 3194-3204.

[6] MelekAcarBoyacioglu, Yakup Kara, Omer KaanBaykan, Predicting bank financial failures using neural networks, support vector machines and multivariate statistical methods: A comparative analysis in the sample of savings deposit insurance fund(SDIF) transferred banks in Turkey, Expert Systems with Applications 36(2009) 3355-3366.

[7] M. Aoba, T. Kikuchi, Y. Takefuzi, Euro banknote recognition system using a three layered perceptron and rbf networks, IPSJ Transactions on mathematical modelling and its applications, vol.44(may 2003) No. SIG 7(TOM 8). Machine learning and cybernetics,22602264.

[8] Ligang Zhou, Performance of corporate bankruptcy prediction models on imbalanced dataset. The effect of sampling methods, Knowledge-Based Systems 41(2013) 16-25.

[9] Qi Yu, YoanMiche, Eric Severin, Amaury Lendasse, Bankruptcy prediction using extreme learning machine and financial expertise, Neurocomputing 128 (2014)296-302.

[10] GuangliNie, Wei Rowe, Lingling Zhang, Yingjie Tian, Yong Shi, Credit card churn forecasting by logistic regression and decision tree, Expert System with Application 38(2011) 15273-15285.

[11] https://www.kaggle.com/ritesaluja/banknote-authentication-uci- dataaccessed on $(5 / 5 / 2020)$.

[12] Guoqiang Zhang, Michael Y. Hu, B. Eddy Patuwo, Daniel C. Indro, Artificial neural networks in bankruptcy prediction: General framework and cross-validation analysis, European Journal of Operation Research 116 (1999) 16-32.

[13] Gaikwad, Mayadevi A., Vaijinath V. Bhosle, and Vaibhav D. Patil., "Automatic Indian New Fake Currency Detection.",International Journal of Engineering Research \& Technology (IJERT), Vol. 6, Issue 11, November, 2017.

[14] Renuka Nagpure, Shreya Sheety, Trupti Ghotkar, "CurrencyRecognition and Fake Note Detection", IJIRCCE, vol. 4, 2016.

[15] M. Deborah and SoniyaPrathap -Detection of Fake currency usingImage Processing. IJISET- International Journal of InnovativeScience, Engineering \& Technology, Vol. 1, Issue 10, 2014.

[16] Faiz M. Hasanuzzaman, Xiaodong Yang, and YingLi Tian, SeniorMember, IEEE Robust and Effective Component-based BanknoteRecognition for the Blind IEEE Trans Syst Man Cybern C ApplRev. 2012 Nov; 42(6): 1021-1030.

[17] Mohammad H Alshayeji, Mohammad AlRousanandDunyaT.Hassoun, Detection Method for Counterfeit Currency Based on Bit Plane Slicing Technique, International Journal of Multimedia andUbiquitous Engineering Vol.10, No.11 (2015).

[18] Komal Vora, Ami Shah, Jay Mehta, A Review Paper on CurrencyRecognition System, International Journal of ComputerApplications (0975 - 8887) Volume 115 - No. 20, April 2015.

[19] Ankush Singh, Prof. Ketaki Bhoyar, Ankur Pandey, Prashant Mankani, Aman Tekriwal Detection of Fake Currency using Image Processing, IJERT, vol. 8, Issue 12, 2019.

[20] Devid Kumar, Surendra Chauhan - Indian Fake Currency Detection using Computer Vision, IRJET, vol. 7. Issue 5, 2020. 
[21] M. Laavanya, V. Vijayaraghavan- Real Time Fake Currency Note Detection using Deep Learning, IJEAT, vol. 9, Issue 1S5, 2019.

[22] Navya Krishna G, Sai Pooja G, Naga Sri Ram B, Yamini Radha V, Rajarajeshwari PRecognition of Fake Currency Note using Convolutional Neural Networks, IJITEE, vol. 8, Issue 5, 2019, 2278-3075.

[23] Dr. P. Mangayarkarasi, Akhilendu, Anakha A S, Meghashree K, Faris A B - Fake Indian Currency Note Recognition, IRJET, vol. 7, Issue 5, 2020.

[24] Snehlata, Vipin Saxena - An Efficient Technique for Detection of Fake Currency, IJRTE, vol. 8, Issue 3, 2019, 2277-3878.

[25] P. Gayathri, P. Soniya, V. YaminiPriya, G. Srinivas, V. Ravindra - Texture Classification for Fake Indian Currency Detection, IJERT, vol. 9, Issue 6, 2020, 2278-0181. 


\title{
Ticket Analytics by an AMS Team to Assess Applications Deficiencies
}

\author{
Biswadeb Bandyopadhyay \\ Assistant Professor, Department of Computer Science and Engineering \\ University of Engineering and Management, Kolkata \\ E-Mail : biswadeb.bandyopadhyay@uem.edu.in \\ Mobile: +91-9903052768
}

\section{Introduction}

The paper discusses a Ticket Analytics undertaken by an Application Maintenance and Support (AMS) team engaged in providing end to end Application Maintenance and Support services of large number of applications performing a host of operational, tactical and strategic functions of the organization. The team has been providing AMS support of these applications for the last two years very efficiently with almost $100 \%$ compliances of all applicable SLAs. The team gained considerable knowledge of the applications and have been credited with solving some of the most critical and challenging production problems. Client has expressed their deep appreciation of the work being done by the AMS team from time to time.

However, client has of late expressed a desire to know more about nature of production incidents and have a better understanding of these applications in terms of their nature of defects to unearth certain fundamental characteristics of these applications. Client therefore wanted the AMS team to delve into the defects that have uncovered so far and carry out a comprehensive analysis of those defects (production incidents) to bring out certain very fundamental characteristics of those applications in terms of nature of future problems they may throw up in their operational life. Idea was to undertake a comprehensive analytics of the production tickets that were created in those applications and come up with a set of predictions of problems those applications may throw up in future. With this knowledge in place, client in addition wanted the team to make suitable recommendations to alleviate those future problems by initiating appropriate corrective and preventive measures.

\section{Client Expectation from AMS Team}

Based on the above client ask, a comprehensive ticket analytics was carried out by the AMS team.

Client had the following questions, which they wanted AMS team to find an answer based on analytics of all the production tickets uncovered so far.

1. A view on ticket volumes, SLA performance and MTTR portfolio wise

2. A view on ticket volumes, SLA performance and MTTR applications wise

3. A view on ticket volumes, SLA performance and MTTR defect classification wise

4. A view on the systems which are in trouble today

5. Predict which applications may have SLA misses in future?

6. Which systems will be required to have predictive maintenance to improve their performance?

7. Which systems appear to be heading for a meltdown (applications which can break easily)?

8. Which systems am I being lazy about and haven't shown improvement for years (applications which need special attention, which probably is not being paid)?

The study has undertaken with production incidents (ticket data), for immediately preceding one year which translated to 1998 incidents that occurred in the span of immediately preceding one year.

There exist 138 individual applications supporting various operational, tactical and strategic requirements of the organization. These applications are classified into 4 portfolios depending on the area of usage and technology used for these applications namely

- GEN-CSL-3 : Group of applications hosted on GenApps technology 
- GEN-CSL-2 : Group of applications hosted on GenApps technology

- SAP-CSL-3 : Group of applications hosted on SAP technology

- SAP-CSL-2 : Group of applications hosted on SAP technology

Objective of this ticket analytics was to look at and analyze production tickets originating in these four portfolios and gain an understanding on critical attributes of these incidents. The critical attributes of interest comprised of the following.

- Ticket Volume

- SLA Performance

- MTTR (Mean Time to Restore)

AMS Team focused on a detailed analysis of the incidents and creating suitable graphical presentation representing each area of concern stated above. AMS team arrived at appropriate problem specifications using a set of hypothesis.

\section{Detailed Technical Approach}

Following section provides a description of the detailed ticket analytics performed by the AMS team with production incidents uncovered in preceding one year.

\section{1 $\quad$ Following section provides} a view on ticket volume, SLA performance and MTTR portfolio wise and Application Wise Ticket Volume
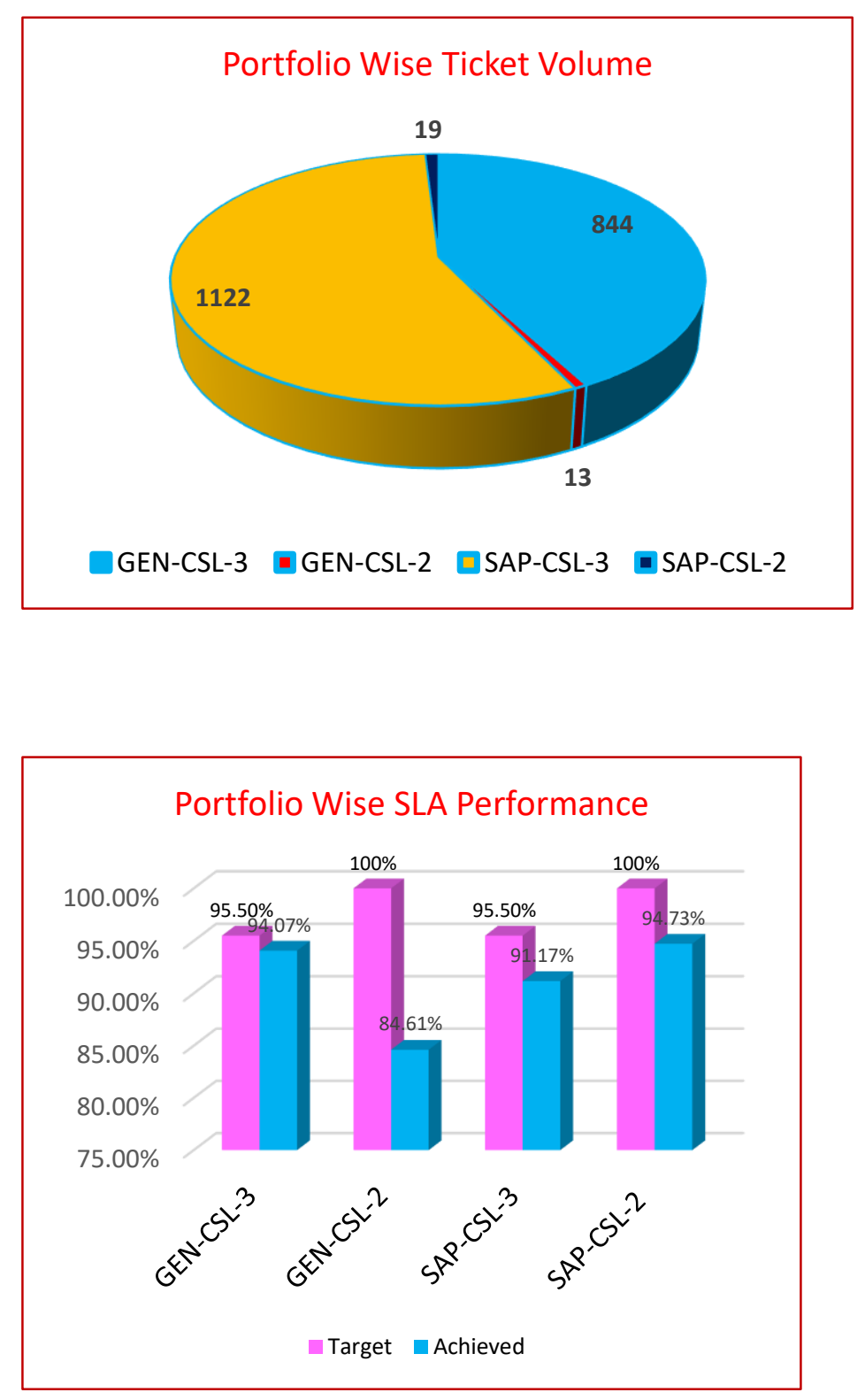


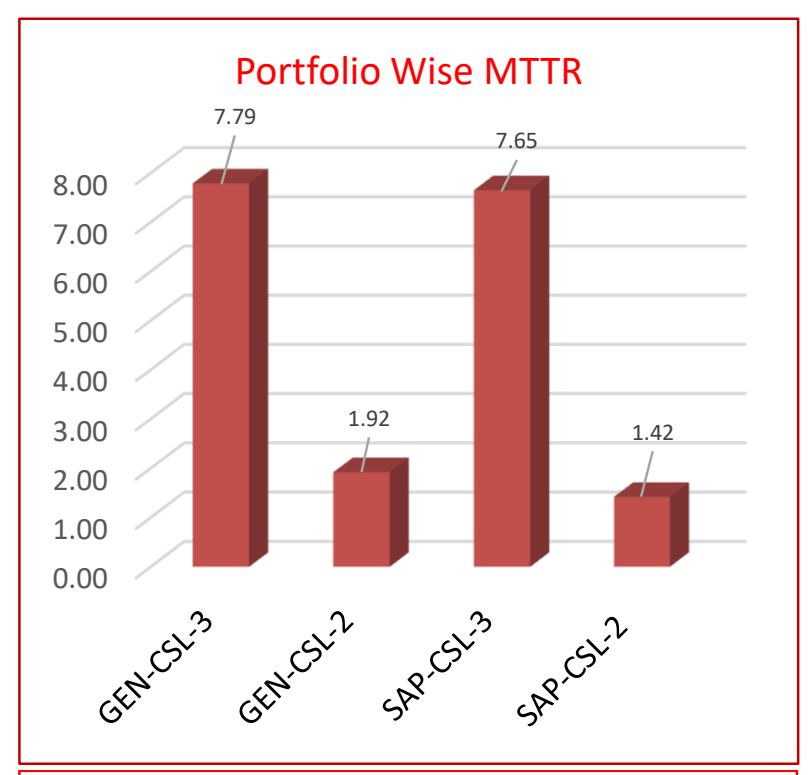

\section{Application Wise Ticket Volumes}
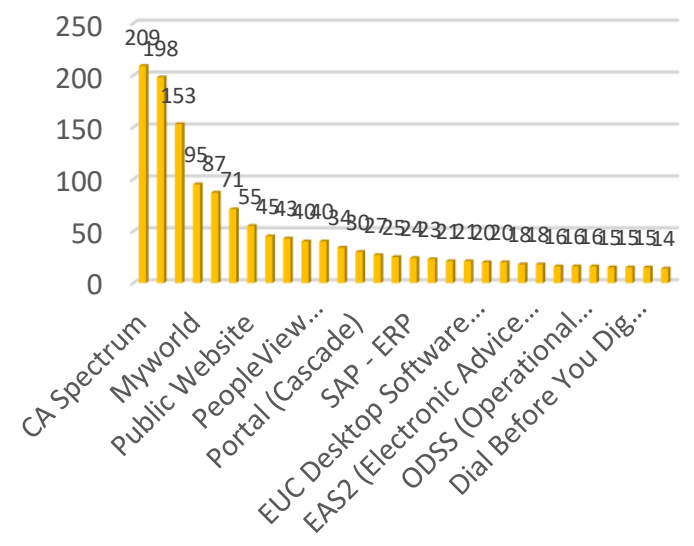

3.2 Following section provides a view on ticket volumes, SLA performance and MTTR applications wise

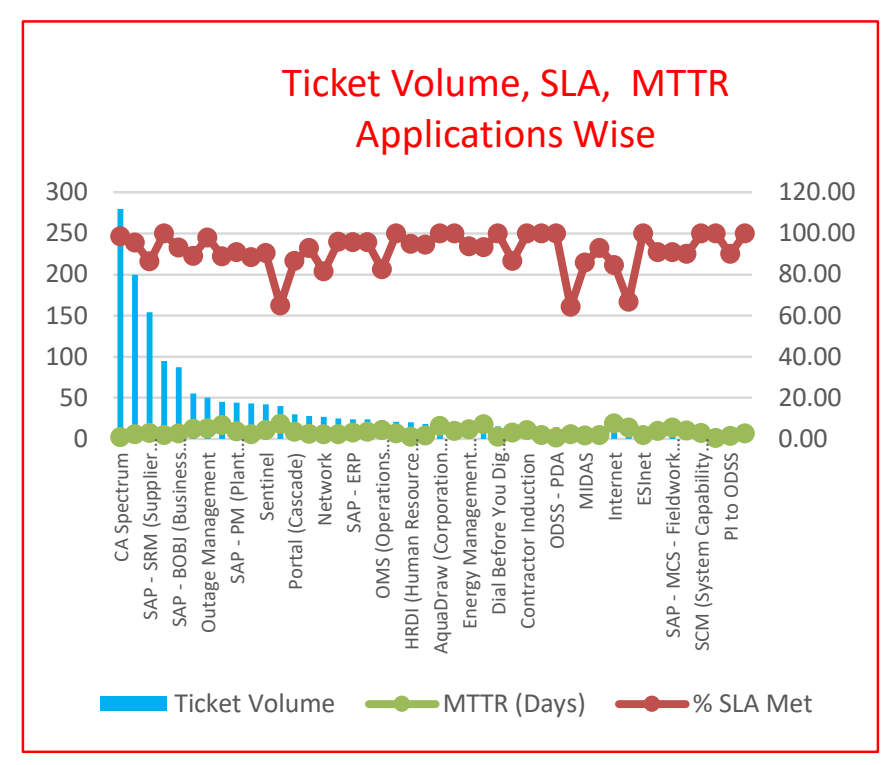

\subsection{Following section provides a view on ticket volumes, SLA performance and MTTR defect classification wise}

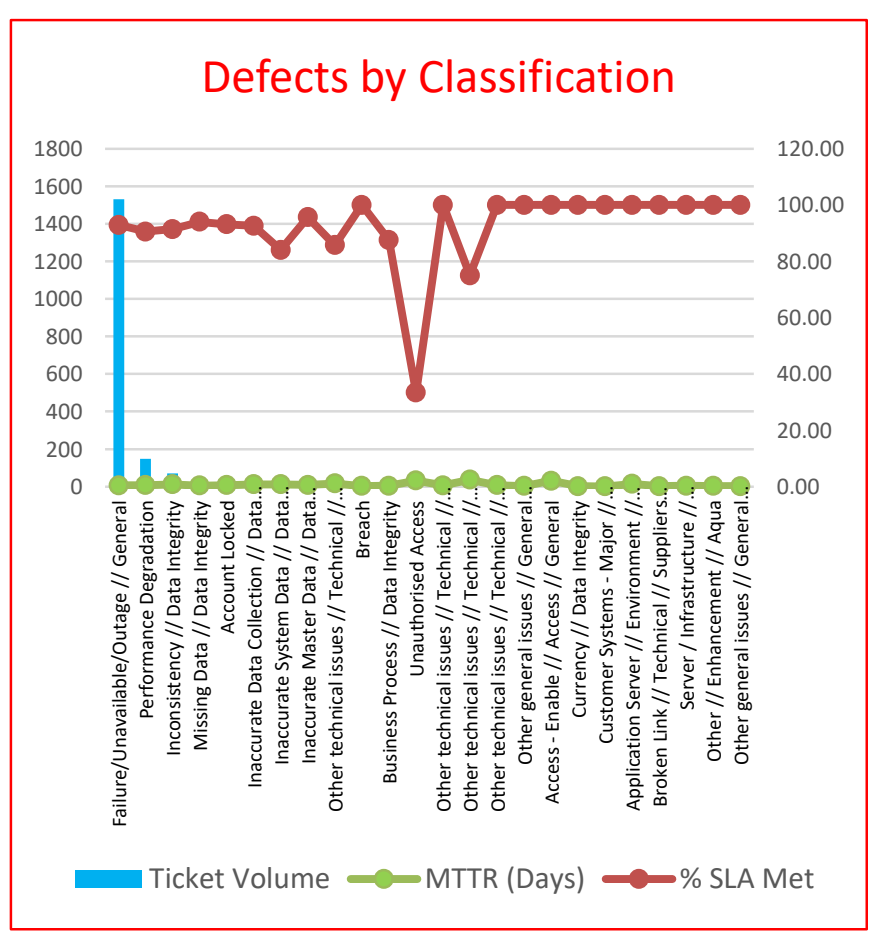




\subsection{A view on the systems which are in trouble today}

Following section provides a list of systems (applications) which are in trouble today. The systems which had SLA misses in the recent past are the systems which are in trouble today.

Diagram below provides the systems which had SLA misses in recent past and are the systems which are in trouble at present. Count against the applications indicates number of SLA misses.

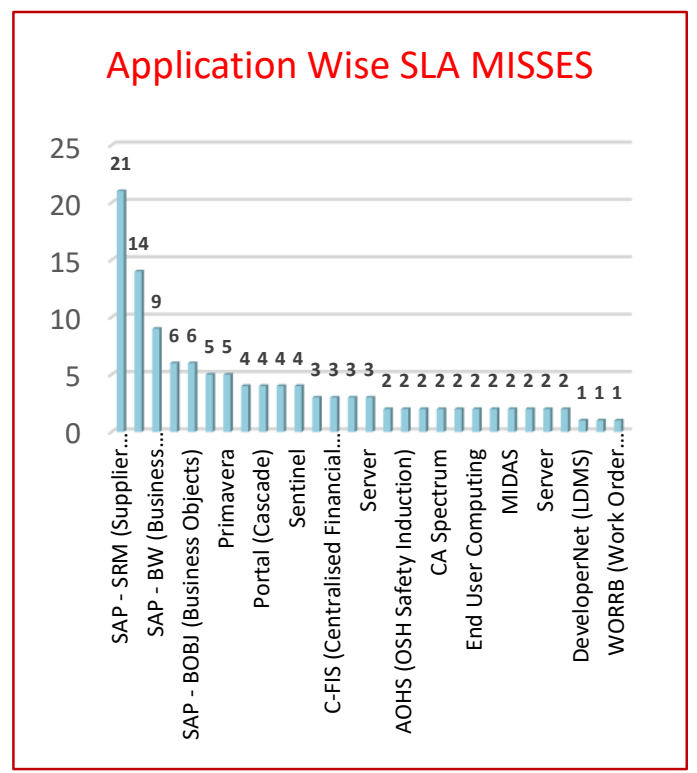

\subsection{Predict which applications may have SLA misses in future?}

Hypothesis used: Applications with higher SLA MISSES in the immediate past will have higher probability of SLA misses in immediate future.

Therefore applications with predicted SLA misses in immediate future are given below. 1 is the highest probability of SLA misses and 0 is the lowest probability of SLA miss as pictorially depicted below.

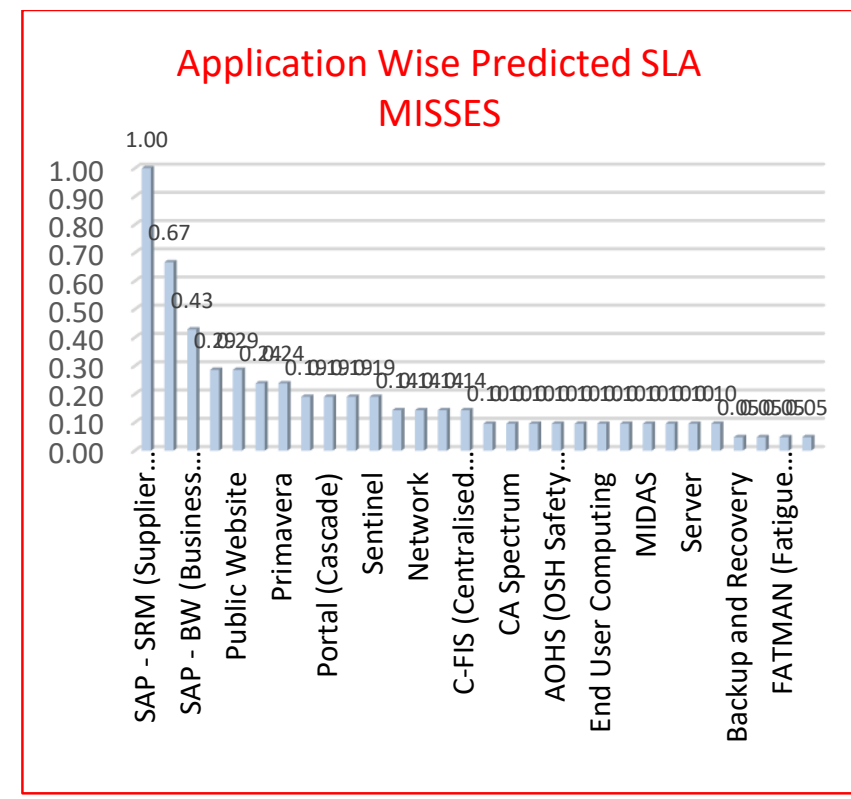

\subsection{Which systems will be required to have predictive maintenance to alleviate their future performance?}

Hypothesis used: Applications with higher ticket volumes in the immediate past will have higher probability of ticket volume in immediate future and will require predictive maintenance. 1 is the highest probability of ticket volume and 0 is the lowest probability of predictive maintenance requirements as pictorially depicted below.

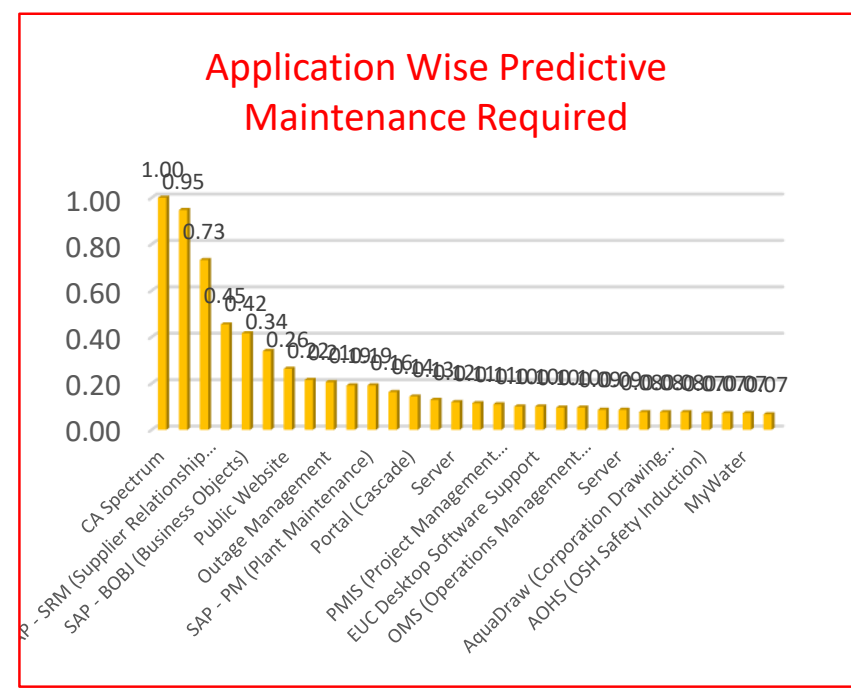




\subsection{Which systems appear to be heading for a meltdown (applications which can break easily)?}

Hypothesis used: Applications with higher Number of Tickets and Higher MTTR in the immediate past are the applications which are heading for meltdown (applications which can break easily). 1 is the highest probability of applications and 0 is the lowest probability applications heading for meltdown depicted in the graph below.

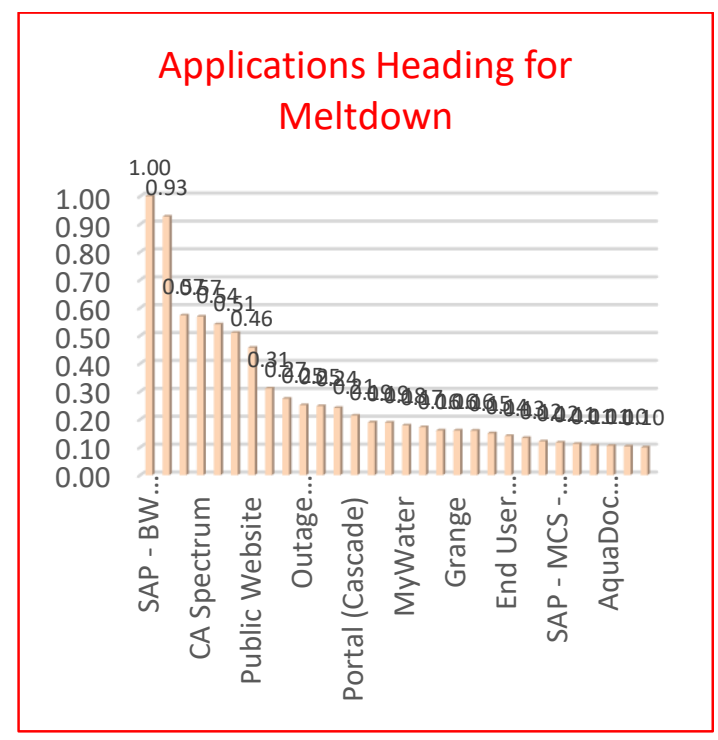

\subsection{Which systems am I being} lazy about and haven't shown improvement for years (applications which need special attention, which probably is not being paid)?

Hypothesis used: Applications with lower Number of Tickets and Higher MTTR in the immediate past are the applications not showing improvements for year. 1 is the highest probability of applications and 0 is the lowest probability of applications not showing improvements for years.

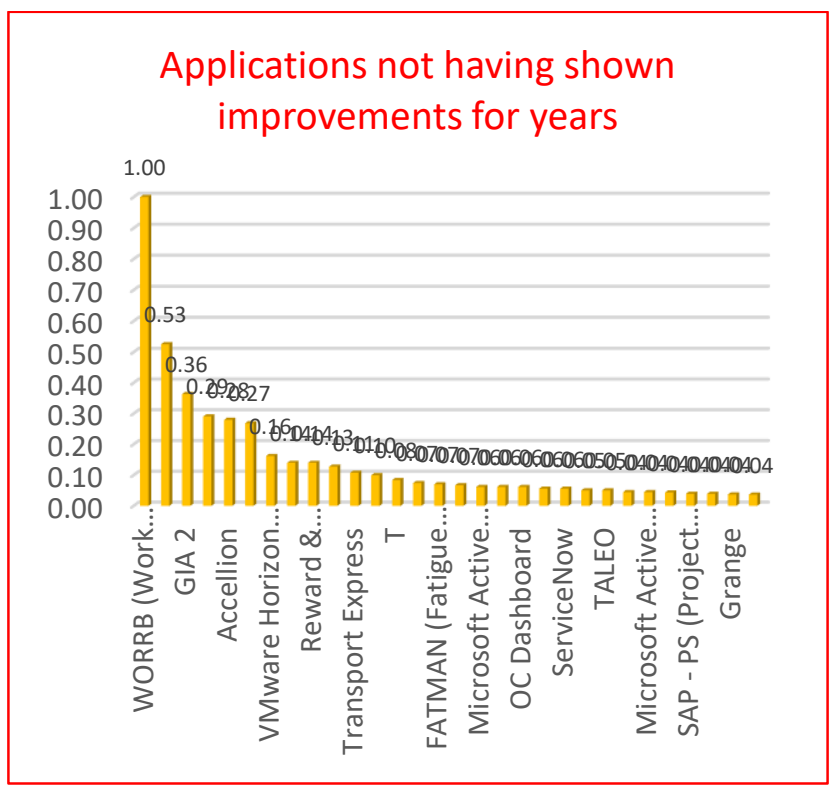

\section{Additional Statistics}

Some additional statistics were created by the team to provide added insight to the client organization about the nature of problems the systems are facing. 


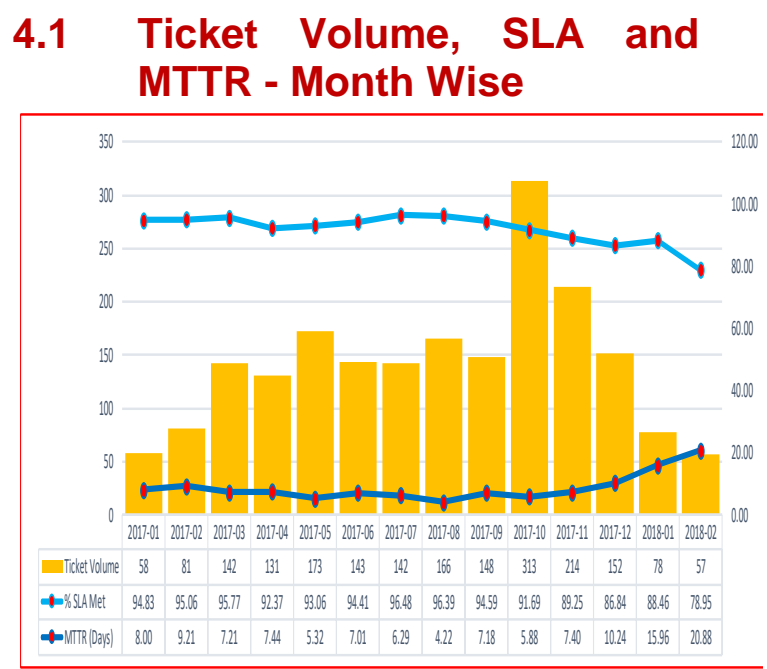

\section{Observations:}

MTTR: Trend is increasing

SLA: Trend dis dedlining

Ticket Voume: Trend is increasing, and then declining since October 2017

\section{SIATargets}

GEN-CSL-2:10\%

GEN-CSL-3: $95.50 \%$

SAP.CLI-2:100\%

SAP.CSL:3:95.50\%

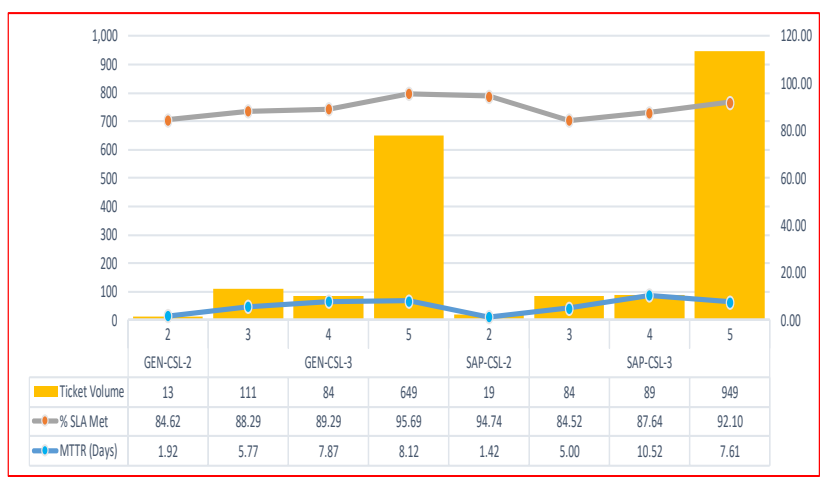

\section{Observations}

MTTR:High in GEN-SS-3 and SAP-CSL-3, Priorities 4 and 5 specifically SLA: Faling across all 4 groups, except GEN-CSL-3 Priority 5 Ticket volume: Highestin Priority 5

SLA Targets GEN-CSL-2: $100 \%$ GEN-CSL-3: $95.50 \%$ SAP-CSL-2: $100 \%$ SAP-CSL-3: $95.50 \%$

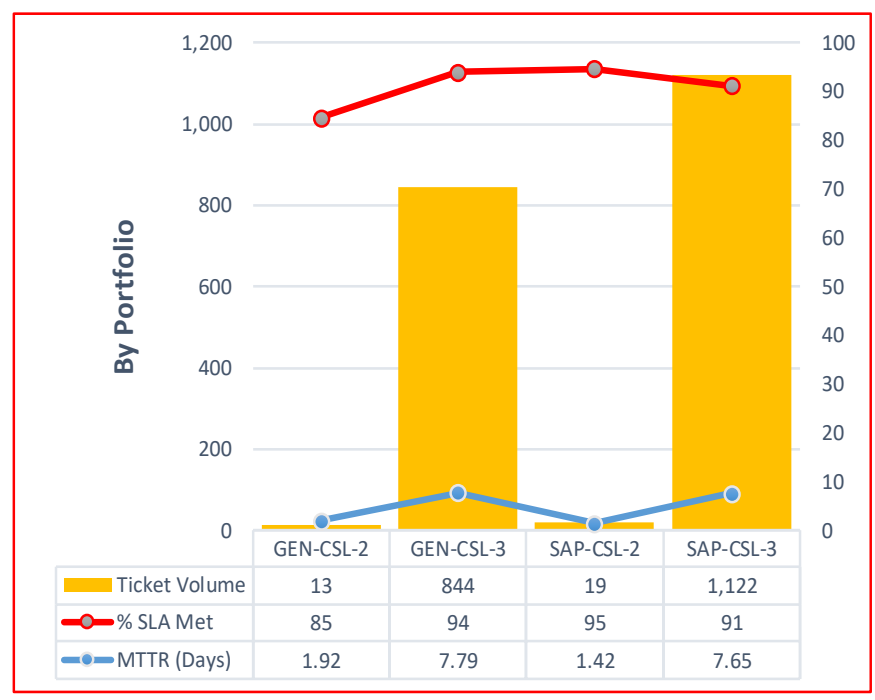

Observations:

MTTR: Low in GEN-CSL-2 and SAP-CSL-2

SLA: Lowest in GEN-CSL-2, but failing across all 4 groups

Ticket volume: Highest in SAP-CSL-3

SLA Targets

GEN-CSL-2: $100 \%$

GEN-CSL-3: 95.50\%

SAP-CSL-2: $100 \%$

SAP-CSL-3: $95.50 \%$

\subsection{Ticket Volume, SLA and MTTR - Portfolio/Priority Wise}




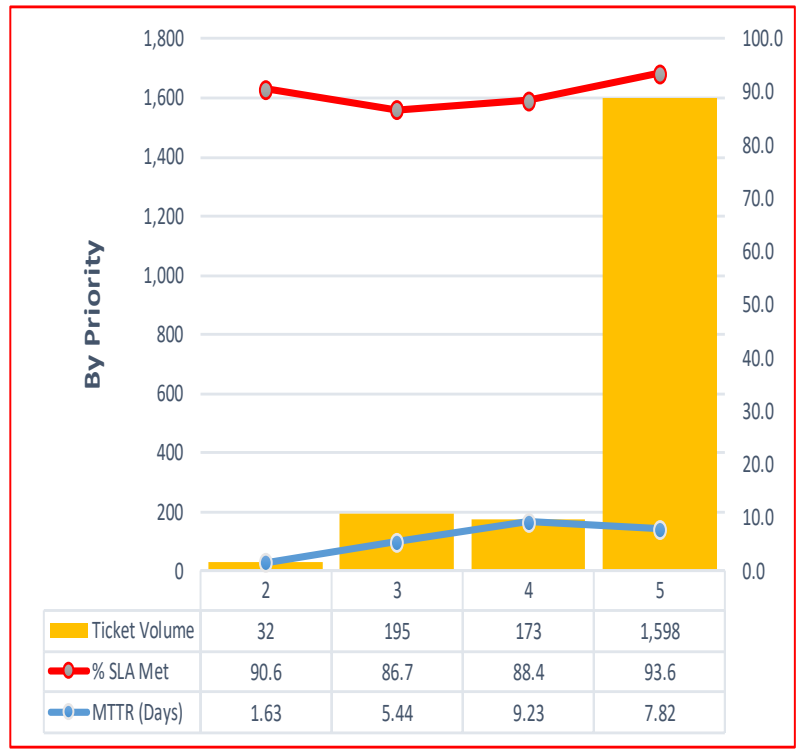

Observations:

MTTR: High for Priorities 4 and Priority 5

SLA: Lowest in P3, but failing across all Priorities

Ticket volume: Highest in Priority 5

SLA Targets

GEN-CSL-2: $100 \%$

GEN-CSL-3: $95.50 \%$

SAP-CSL-2: $100 \%$

SAP-CSL-3: $95.50 \%$

\subsection{Ticket Volume, SLA and MTTR - Application Wise}

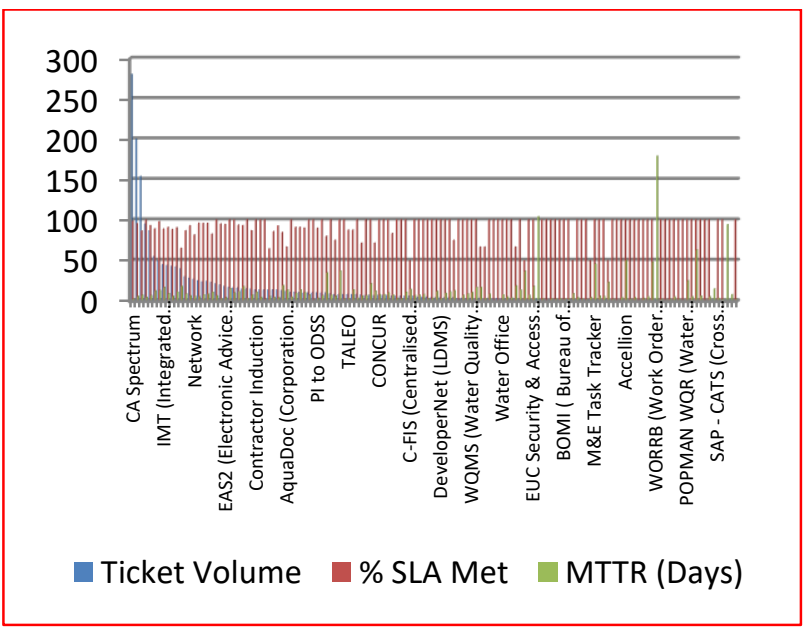

\section{Recommendations}

Based on above detailed ticket analytics the AMS team made following recommendations to the client organization. Recommendations were identified highlighting any gaps in applicable process and process implementation.

- Study the applications which are in trouble. Look at the root cause of those tickets attributed to SLA misses and have following action plan in place

$>$ Lack of application knowledge Identify resource skill matrix, training plan, develop training modules, application knowledge repository, resource wise training calendar.

$>$ Lack of business knowledge - Arrange periodic training sessions by Business Analyst imparting business and industry knowledge to the AMS team as appropriate.

> Lack of SME support - Identify SME support to bridge the knowledge gaps, focused and systematic training to reduce skill gaps and industry knowledge.

$>$ Dependency issue (Business, Technical) - Advance planning, regular governance meeting with all stake holders, highlighting any dependency with advance provisioning.

$>$ Deliverable quality issue (inadequate review, testing) - Look at review and test plan, review and test cycle time, review and test coverage and efficiency, establish end to end test metrics highlighting any gaps in review and testing process.

$>$ Infrastructure issue or environmental issue - Regular multi vendor governance, discuss and plan any infrastructure issue or environmental requirements, ensure advance intimation and provisioning.

> Test environment/test data issue Regular governance with test team, review any test environment/test data issues, review any known constraints, put in place and advance remedial plan for ensuring environment, test data availability.

> Lack of automated testing, regression testing - Explore automated unit testing, regression test, advance test data preparation

- Study the application which may be in trouble. Analyze historical SLA misses in those applications. Look at the root cause of those 
tickets attributed to SLA misses and have similar plan as above in place.

- Look at systems which are contributing to highest volume of tickets, and bring down the number of tickets by way of process improvements, looking at Classification of failures by identifying/improving any generic/recurring cause of failures.

- $\quad$ Perform RCA on applications predicted to result in SLA misses

- Focus on and evolve a clear action plan for applications with Predictive Maintenance Required for these Applications

- Bring to Management and IT attention for Systems identified as heading for meltdown and Systems being lazy about, and put in place a corrective and recovery action plan

- Bring down the high Mean Time to Resolve (MTTR) of P3,P4,P4 by way of additional knowledge acquisition, on the job training

- Look at systems which are contributing to highest volume of tickets, and bring down the number of tickets by way of process improvements, looking at Classification of failures by identifying/improving any generic/recurring cause of failures.

- Perform a detailed analysis on application wise SLA Misses. Perform a detailed analysis on the applications with SLAs misses, defect classification of the tickets, MTTR of tickets, system environment, test environment, review and testing done. Based on this analysis, attributed the SLA misses to following set of possible reasons. The reasons include lack of application knowledge, lack of business knowledge, infrastructure issue, environmental issue, system load issue, SME support issue, resource issue, any constraints/limitations. Based on this analysis tailor an action plan to remedy the cause of the SLA misses which could include Analyze applications with predicted SLA misses. Strengthen current knowledge level of the team around those application areas, look at historical SLA misses from the above analysis prepare and put in place a set of action plans to alleviate any constraints/limitation that may impact the SLA adherence. Develop training plans and modules, resource re-alignment as appropriate, upfront plan for SME availability, system availability, test environment, test data, and introduce COG3 for ticket resolution.
- Similar Action Plan should be formulated as above for applications with Predictive Maintenance Required.

- Bring to Management and IT attention for systems identified as heading for meltdown and systems being lazy about, and put in place a corrective and recovery action plan. This would include analyzing the business impact of such meltdown, ability of the team to quickly address critical issues and have an action plan in place for any anticipated surge in ticket volumes. This may include resource augmentation, resource realignment, ensuring SME support, ensuring system availability, test data availability, with eventually heightened focus around these applications.

- $\quad$ Carry out a similar root cause analysis on high MTTR in P3, P4,P5 tickets, and identify a set of possible causes namely additional knowledge acquisition, on the job training, right skilling acquisition, SME knowledge acquisition, system/infrastructure availability issue, test environment/test data issue, etc. This would be followed by a similar action plan as above, for improvements in all above areas in a time bound manner.

- Introduce COG3 for improving Mean Time to Resolve (MTTR) for tickets of all classes

\section{Conclusion}

As mentioned in the beginning, that the Application Maintenance and Support (AMS) team was engaged in providing end to end Application Maintenance and Support services of large number of applications performing a host of operational, tactical and strategic functions of the organization. The team has been providing AMS support of these applications for the last two years very efficiently with almost $100 \%$ compliances of all applicable SLAs. The team gained considerable knowledge of the applications in the process and have been able to solve some of the most critical and challenging production problems. Client has expressed their deep appreciation of the work being done by the AMS team from time to time.

However, in spite of the best effort by this AMS team, it was found that, there was still fairly large number of production incidents that were being opened. In view of the same, client wanted to get to the bottom of the problem and wanted a better understanding on these applications in terms of 
their nature of defects to unearth certain fundamental characteristics of these applications. Client therefore wanted the AMS team to delve into the defects that have uncovered so far and carry out a comprehensive analysis of those defects (production incidents) to bring out certain very fundamental characteristics of those applications in terms of nature of future problems they may throw up in their operational life. Idea was to undertake a comprehensive analytics of the production tickets that were created in those applications and come up with a set of predictions of problems those applications are likely throw up in future. With this knowledge in place, client in addition wanted the team to make suitable recommendations to alleviate those future problems by initiating appropriate corrective and preventive measures. The AMS team focused on this ticket analytics and made the above recommendations a series of steps which will have potential impact on those applications and will bring down the number of production incidents originating from those applications.

\section{Key References and Bibliography}

1. Incident Ticket Analytics for IT Application Management Services by Ta-Hsin Li, Rong Liu, Noi Sukaviriya, Ying Li, Jeaha Yang, Michael Sandin, and Juhnyoung Lee IBM T.J. Watson Research Center Yorktown Heights, NY 10598-0218, USA

2. Incident Ticket Analytics for IT Application Management Services by R. Liu, 2014 IEEE International Conference on Services Computing

3. Significance of Ticket Analytics in Effective Software Maintenance: Awareness by Sharon Christa Dept. of Information Science and Engineering, DSCE, Bangalore

4. STAR: A System for Ticket Analysis and Resolution by Wubai Zhou, Florida International University, USA 
